Japan. J. Math.

Vol. 28, No. 2, 2002

\title{
Maslov index in the infinite dimension and a splitting formula for a spectral flow
}

\author{
By Kenro Furutani and Nobukazu Otsuki \\ (Received April 13, 2001) \\ (from Tokyo Journal of Mathematics)
}

\begin{abstract}
First, we prove a local spectral flow formula (Theorem 3.7) for a differentiable curve of selfadjoint Fredholm operators. This formula enables us to prove in a simple way a general spectral flow formula (Theorem 3.8) which was already proved in [BF1]. Secondly, we prove a splitting formula (Theorem 4.12) for the spectral flow of a curve of selfadjoint elliptic operators on a closed manifold, which we decompose into two parts with commom boundary. Then the formula says that the spectral flow is a sum of two spectral flows on each part of the separated manifold with naturally introduced elliptic boundary conditions. In the course of proving this formula, we investigate a property of the Maslov index for paths of Fredholm pairs of Lagrangian subspaces.
\end{abstract}

1. Introduction

2. Maslov index in the infinite dimension

2.1. Fredholm-Lagrangian-Grassmannian

2.2. Maslov index for paths

2.3. Maslov index for the curve of Fredholm pairs of Lagrangian subspaces

3. Spectral flow formula

3.1. Symmetric operators and boundary value space

3.2. Definition of the spectral flow

3.3. Local spectral flow formula

3.4. General spectral flow formula

4. Splitting formula for a spectral flow

4.1. Splitting of manifolds and first order differential operators

4.2. A reduction formula for the Maslov index

4.3. Splitting formula for a spectral flow

Appendix

References

1991 Mathematics Subject Classification. 32C17, 57R15, 58F06.

Key words and Phrases. Maslov index, spectral flow, Fredholm-Lagrangian-Grassmannian, elliptic operator, K-group, unitary group. 


\section{Introduction}

It is well known that the non-trivial component $\widehat{\mathcal{F}}_{*}$ of the space $\widehat{\mathcal{F}}=\widehat{\mathcal{F}}_{-} \cup$ $\widehat{\mathcal{F}}_{*} \cup \widehat{\mathcal{F}}_{+}$of bounded selfadjoint Fredholm operators (on a complex or a real Hilbert space) is a classifying space for the $K$-group, $K^{-1}$ in the complex case and that of $\mathrm{KO}^{-7}$ in the real case ([AS]). Also we know, by Bott periodicity theorem, that both of the fundamental groups of these spaces is isomorphic to $\mathbb{Z}$. This isomorphism is given by the quantity "spectral flow", although it is not stated explicitly in this paper [AS]. We have an "intuitive" understanding of the spectral flow by saying that the spectral flow is the difference of the "net numbers" of the eigenvalues of the selfadjoint Fredholm operators which change signs from minus to plus and from plus to minus, when the parameter of the family goes from 0 to 1 .

Since the "spectral flow" was treated in the paper [APS], the quantity "spectral flow" appears in the various theories where it plays important roles, for example, in the study of spectral analysis of Dirac operators, in the theory of Floer homology for the study of low dimensional manifolds, in the study of symplectic topology and so on.

Then in the paper $[\mathrm{Ph}]$, a rigorous definition of the spectral flow was given for not only continuous loops in the space $\widehat{\mathcal{F}}_{*}$ but also for arbitrary continuous paths in $\widehat{\mathcal{F}}_{*}$ and it was proved that the quantity is a homotopy invariant of the continuous path in the space $\widehat{\mathcal{F}}_{*}$ with the fixed end points and satisfies the additivity under catenation of the continuous paths. Thus the spectral flow is not only a spectral invariant but also a homotopy invariant, in so far as we can treat it in the framework of the space $\widehat{\mathcal{F}}_{*}$.

Nowadays there are many types of formulas including various "spectral flows" corresponding to families of Fredholm operators which are mostly unbounded operators, because simply they are families of differential operators. However in some cases the "continuous" family of such unbounded Fredholm operators can be interpreted as a continuous path in the space $\widehat{\mathcal{F}}_{*}$ as it was treated in the papers [FO], $[\mathrm{BF} 1]$ and $[\mathrm{CP}]$. Also the theorems in $[\mathrm{Fl}],[\mathrm{Yo}]$ and $[\mathrm{Ni}]$ can be interpreted in the framework of the space $\widehat{\mathcal{F}}_{*}$. See also [Ta], [Ge], [CLM2], [OF] and [DK] and others.

It is not clear for the present authors whether arbitrary "continuous" families of Fredholm operators, especially a family of unbounded selfadjoint Fredholm operators with varying domains of definitions, can be interpreted in the framework of the space $\widehat{\mathcal{F}}_{*}$.

The main purpose of this paper is to prove a general splitting formula (Theorem 4.12) for the spectral flow of a family $\left\{A+C_{t}\right\}_{t \in[0,1]}\left(C_{t}\right.$ is of zeroth order) of first order selfadjoint elliptic differential operators on a closed manifold in the framework of the space $\widehat{\mathcal{F}}_{*}$. When we decompose the closed manifold $M$ into two parts $M_{ \pm}$by a hypersurface $\Sigma, M=M_{-} \cup M_{+}, M_{-} \cap M_{+}=\partial M_{ \pm}=\Sigma$, then we also have a family of symmetric elliptic operators on each part $M_{ \pm}$simply by 
the restriction, and at this level it is nothing more than having two families of symmetric operators defined on manifolds with boundary. This means there are no natural choices of boundary conditions among selfadjoint elliptic boundary conditions under which the family becomes a family of selfadjoint operators. Under these circumstances it will not be apparent whether the spectral flow of the family $\left\{A+C_{t}\right\}$ on the whole manifold is expressed as a sum of two spectral flows obtained by even imposing a "suitable" selfadjoint elliptic boundary condition on each part and adding a correction term which solely depends on the boundary manifold $\Sigma$, partly for the reason that we have no integral representations of the spectral flow on $M$.

To formulate our splitting formula in this situation, we shall take the following way, that is, firstly we construct a two-parameter family $\left\{\mathcal{A}_{s, t}\right\}_{s, t \in[0,1]}$ of selfadjoint Fredholm operators on $M$ with $\mathcal{A}_{t, t}=A+C_{t}$. Then by noting the homotopy invariance and the additivity under catenation of the spectral flow, we will see that the sum of two spectral flows of $\left\{\mathcal{A}_{s, 0}\right\}_{s \in[0,1]}$ and $\left\{\mathcal{A}_{1, t}\right\}_{t \in[0,1]}$ coincides with the spectral flow of the original family (Theorem 4.4). From the definition (see (4.1)), it looks as if the families $\left\{\mathcal{A}_{s, 0}\right\}_{s \in[0,1]}$ and $\left\{\mathcal{A}_{1, t}\right\}_{t \in[0,1]}$ are defined on each component respectively; however in general it is not clear whether each of these two spectral flows coincides with a spectral flow of a family obtained by imposing a suitable selfadjoint elliptic boundary condition on the restriction of $\left\{A+C_{t}\right\}$ to each part. Then secondly we prove that if the operators in the family are of a product form near the separating hypersurface, then there is a selfadjoint elliptic boundary condition on each part under which the restrictions of the operators to each part become a family of selfadjoint Fredholm operators and their spectral flows coincide with each of $\left\{\mathcal{A}_{s, 0}\right\}_{s \in[0,1]}$ and $\left\{\mathcal{A}_{1, t}\right\}_{t \in[0,1]}$ (Theorem 4.12). Moreover it will turn out that these boundary conditions reflect the influence from one side to other side of $M_{ \pm}$in a natural way. We verify these completely in the framework of the space $\widehat{\mathcal{F}}_{*}$. Our general spectral flow formula (Theorem 3.8) and a reduction theorem (Theorem 4.5) play a role of a bridge connecting these two spectral flows for the family of operators of a product form near the separating hypersurface $\Sigma$.

There are several similar formulas already treated in the papers [Ta], [DK], [CLM2] and others. Here we would like to emphasize that we can admit noninvertible end points in the family $\left\{A+C_{t}\right\}$ because we base our arguments on the rigorous definition of spectral flow as a homotopy invariant of the spectral flow given in $[\mathrm{Ph}]$ together with that of the Malsov index which is valid without any assumptions at the end points. Our method will explain to some extent what kinds of conditions to the operators in the family we need to prove such a splitting formula. Moreover, since for a family of the operators with product form structure near the separating hypersurface, the space of our boundary values $\beta=D_{\max } / D_{\min }$ is identical for any length of the "neck", we expect this property also will give us a reduction of our splitting formula (Theorem 3.8) under taking adiabatic limits 
within our framework.

The contents of this paper are as follows.

In the paper [BF1] a theory was developed for the Maslov index in the infinite dimensional case. However there we based our arguments on an erroneous characterization of the Fredholm pair of Lagrangian subspaces given in [BW], which led us to introduce a small space of Fredholm-Lagrangian-Grassmannian. Although we corrected this part in the errata of the preceding paper [BFO], here in $\S 2$ we will develop the investigation again from the very beginning of the space of FredholmLagrangian-Grassmannian and the theory of Maslov index in the infinite dimension. Especially, the difference from the well-known finite dimensional case will be made clear and we study a relation of the Maslov index of the continuous curve of Fredholm pairs of Lagrangian subspaces with respect to the diagonal in the product space of symplectic Hilbert spaces and the Maslov index of a continuous curve with respect to a fixed Lagrangian subspace (Proposition 2.14).

In $\S 3$ we give a new proof of the general spectral flow formula (Theorem 3.8) which was proved in the paper [BF1]. We prove it based on the local spectral flow formula (Theorem 3.7). This new proof will give us a clear understanding of the phenomena surrounding the behavior of the spectrum of selfadjoint Fredholm operators under a certain perturbation.

In $\S 4$ first we review a reduction theorem (Theorem 4.5) of the Maslov index in the infinite dimension which we have proved in the paper [BFO], and in the last section $\S 4.3$ we prove our main Theorem 4.12. Before proving this Theorem we analyze the possibility to express the spectral flow as a sum of spectral flows of two families constructed from the original family of elliptic operators which are not necessarily of a product form near the separating hypersurface $\Sigma$ (Theorem 4.4). From this formula we arrive at our main Theorem under the assumption that the operators in the family are of a product form near the separating hypersurface $\Sigma$. The preceding reduction theorem in $\S 4.2$ of the Maslov index is used twice here, which will explain the role of the product form assumption on the operators near the separating hypersurface $\Sigma$.

Throughout this paper we shall work in the real category, that is, Hilbert spaces are real and elliptic operators are defined on a real vector bundle.

Finally we remark that although there is a deep theory of pseudo-differential operators with transmission property including the theory of Calderón projector ([Ho2]), here we avoid the use of Calderón projector in the $L_{2}$-framework to deal with the Cauchy data space. This is because it will make things confusing to use the Calderón projector from the beginning in the $L_{2}$-framework, and furthermore it will force us to miss the role of the product form assumption on the operators in the splitting formula. However we must employ two facts without proofs from the pseudo-differential operator theory: 
(a) The space of boundary values $\boldsymbol{\beta}=D_{\max } / D_{\min }$ of sections in the maximum domain in $L_{2}$-space of a first order selfadjoint elliptic differential operator on a manifold with boundary $\Sigma$ is included in the Sobolev space of order $-\frac{1}{2}$ on the boundary manifold ([Hol]).

(b) If a first order symmetric elliptic differential operator is of a product form near the boundary manifold (= separating hypersurface in our setting), then the closed extension defined by the Atiyah-Patodi-Singer boundary condition is selfadjoint and satisfy "coercive estimate", which we need to determine the space $\boldsymbol{\beta}$ (Proposition 4.6, also see [APS]).

\section{Maslov index in the infinite dimension}

2.1. Fredholm-Lagrangian-Grassmannian. Let $(H,\langle\cdot, \cdot\rangle, \omega)$ be a symplectic (real and separable) Hilbert space. Here $\langle\cdot, \cdot\rangle$ denotes the inner product and $\omega$ the symplectic form, i.e., a non-degenerate, skew-symmetric bounded bi-linear form.

In the discussion below we do not replace the symplectic form $\omega$ after once it was introduced in the real Hilbert space $H$, but we may always assume that there exists a bounded operator $J: H \rightarrow H$ such that $\omega(x, y)=\langle J x, y\rangle$ for any $x, y \in H$ and $J^{2}=-$ Id by replacing the inner product by another one which defines an equivalent norm on $H$. So we can assume from the beginning the following relations:

$$
\begin{aligned}
& { }^{t} J=-J, \quad\langle J x, J y\rangle=\langle x, y\rangle \quad \text { and } \\
& \omega(J x, J y)=\omega(x, y) \quad \text { for all } x, y \in H .
\end{aligned}
$$

Here ${ }^{t} J$ denotes the transpose of $J$ with respect to the inner product $\langle\cdot, \cdot\rangle$.

Let $\mathcal{L}(H)$ denotes the set of all Lagrangian subspaces of $H$.

Each Lagrangian subspace is closed, and the topology on $\mathcal{L}(H)$ is given by embedding it into the space $\mathcal{B}(H)$ of bounded linear operators on $H$ by identifying each Lagrangian subspace with the orthogonal projection onto it.

We fix a Lagrangian subspace $\lambda \in \mathcal{L}(H)$.

Definition 2.1. (a) The Fredholm Lagrangian Grassmannian of $H$ with respect to $\lambda$ is defined as

$$
\mathcal{F} \mathcal{L}_{\lambda}(H):=\{\mu \in \mathcal{L} \mid(\mu, \lambda) \text { is a Fredholm pair }\}
$$

(b) We call the subset

$$
\mathfrak{M}_{\lambda}(H):=\left\{\mu \in \mathcal{F L}_{\lambda}(H) \mid \mu \cap \lambda \neq\{0\}\right\}
$$

the Maslov cycle with respect to $\lambda$. 
Note that a pair $(\mu, \lambda)$ of closed subspaces is called a Fredholm pair, if it satisfies

(1) $\operatorname{dim} \mu \cap \lambda<+\infty$,

(2) $\mu+\lambda$ is closed and $\operatorname{dim} H /(\mu+\lambda)<+\infty$.

In our case, i.e., for a Fredholm pair $(\mu, \lambda)$ of Lagrangian subspaces $\operatorname{dim} \lambda \cap \mu=$ $\operatorname{dim} H /(\lambda+\mu)$.

When we regard the real Hilbert space $H$ as a complex Hilbert space by the almost complex structure $J$ with the obvious Hermitian inner product $\langle\cdot, \cdot\rangle_{J}=$ $\langle\cdot, \cdot\rangle-\sqrt{-1} \omega(\cdot, \cdot)$, we denote it by $H_{J}$. Then for a fixed $\lambda \in \mathcal{L}(H)$ we have an identification

$$
\begin{aligned}
H_{J}=\lambda \oplus \lambda^{\perp}= & \lambda \oplus J \lambda \cong \lambda \otimes \mathbb{C} \\
& x+J y \mapsto x \otimes 1+y \otimes \sqrt{-1}
\end{aligned}
$$

with the complex conjugate map $\tau_{\lambda}(x+J(y))=x-J(y), x, y \in \lambda$.

We denote the group of unitary operators on $H_{J}$ by

$$
\mathcal{U}\left(H_{J}\right)=\left\{U \in \mathcal{B}(H) \mid U J=J U \text { and } U^{t} U={ }^{t} U U=\mathrm{Id}\right\} .
$$

We have a natural action of $\mathcal{U}\left(H_{J}\right)$ on $\mathcal{L}(H)$ and so by fixing a $\lambda \in \mathcal{L}(H)$ we have a surjective map $\rho$ :

$$
\begin{aligned}
\rho: \mathcal{U}\left(H_{J}\right) & \rightarrow \mathcal{L}(H) \\
U & \mapsto U\left(\lambda^{\perp}\right)
\end{aligned}
$$

Put $\mathcal{U}_{\lambda}\left(H_{J}\right):=\rho^{-1}\left(\mathcal{F} \mathcal{L}_{\lambda}(H)\right) \subset \mathcal{U}\left(H_{J}\right)$. Then each $U \in \mathcal{U}_{\lambda}\left(H_{\lambda}\right)$ is decomposed into $U=X+\sqrt{-1} Y$, under the the identification (2.1), such that

$$
\begin{aligned}
& X, Y: \lambda \rightarrow \lambda \text { bounded, } \\
& X^{t} Y=Y^{t} X \text { and } X^{t} X+Y^{t} Y=\mathrm{Id}, \\
& X \quad \text { Fredholm operator. }
\end{aligned}
$$

In the same way as for the finite dimensional case ([Le]), we define the map $\mathcal{S}_{\lambda}$

$$
\mathcal{S}_{\lambda}: \mathcal{U}_{\lambda}\left(H_{J}\right) \ni U=X+\sqrt{-1} Y \mapsto W_{\mu}:=(X+\sqrt{-1} Y)\left({ }^{t} X+\sqrt{-1}^{t} Y\right)
$$

where $W_{\mu}$ depends only on $\mu=U\left(\lambda^{\perp}\right)$. Note that for $U=X+\sqrt{-1} Y,{ }^{t} X+$ $\sqrt{-1}^{t} Y=\tau_{\lambda} \circ U^{*} \circ \tau_{\lambda} \equiv \theta_{\lambda}(U)$. So we can write $\mathcal{S}_{\lambda}(\mu)=U \circ \theta_{\lambda}(U)$. 
We can easily check that $W_{\mu}+\operatorname{Id}=2 X\left({ }^{t} X+\sqrt{-1}^{t} Y\right)$ is a Fredholm operator. Also we have for $x, y \in \lambda$ the point $z=x \otimes 1+y \otimes \sqrt{-1} \in H_{J}$ belongs to $\mu$ if and only if $-z=W_{\mu}\left(\tau_{\lambda}(z)\right)$, so

$$
\operatorname{Ker}\left(W_{\mu}+\mathrm{Id}\right)=(\mu \cap \lambda) \otimes \mathbb{C} \cong(\mu \cap \lambda) \oplus J(\mu \cap \lambda) .
$$

Hence

Proposition 2.2. For any $\mu \in \mathcal{F} \mathcal{L}_{\lambda}(H)$ and any $U \in \mathcal{U}_{\lambda}\left(H_{J}\right)$ with $\mu=$ $U\left(\lambda^{\perp}\right)$

$$
\operatorname{dim}_{\mathbb{R}}(\mu \cap \lambda)=\operatorname{dim}_{\mathbb{C}} \operatorname{Ker}\left(W_{\mu}+\mathrm{Id}\right)
$$

Let us now consider the space

$$
\mathcal{U}_{\mathcal{F}}\left(H_{J}\right):=\left\{U \in \mathcal{U}\left(H_{J}\right) \mid U+\mathrm{Id} \text { is a Fredholm operator }\right\}
$$

and a subset

$$
\mathcal{U}_{\mathfrak{M}}\left(H_{J}\right):=\left\{U \in \mathcal{U}_{\mathcal{F}}\left(H_{J}\right) \mid \operatorname{Ker}(U+\mathrm{Id}) \neq\{0\}\right\}
$$

which we can regard as a kind of the universal Maslov cycle, since $\mathcal{S}_{\lambda}^{-1}\left(\mathcal{U}_{\mathfrak{M}}\left(H_{J}\right)\right)=$ $\mathfrak{M}_{\lambda}(H)$ for any $\lambda$.

Now we state the fundamental property for discussing the Maslov index in the infinite dimension.

Proposition 2.3.

$$
\pi_{1}\left(\mathcal{F} \mathcal{L}_{\lambda}(H)\right) \simeq \mathbb{Z}, \quad \pi_{1}\left(\mathcal{U}_{\mathcal{F}}\left(H_{J}\right)\right) \simeq \mathbb{Z}
$$

and the induced map $\left(\mathcal{S}_{\lambda}\right)_{*}: \pi_{1}\left(\mathcal{F} \mathcal{L}_{\lambda}(H)\right) \rightarrow \pi_{1}\left(\mathcal{U}_{\mathcal{F}}\left(H_{J}\right)\right)$ is an isomorphism.

Proof. We know already that $\pi_{1}\left(\mathcal{F} \mathcal{L}_{\lambda}(H)\right) \simeq \mathbb{Z}$. The fact $\pi_{1}\left(\mathcal{U}_{\mathcal{F}}\left(H_{J}\right)\right) \simeq \mathbb{Z}$ is proved in the appendix. There we will explain a more natural meaning of the space $\mathcal{U}_{\mathcal{F}}\left(H_{J}\right)$ and the proof is similar to that of the first fact (see also a recent work by Kirk-Lesch [KL], where a simple proof is given based on the Calkin algebra).

2.2. Maslov index for paths. In this subsection we review the functional analytic definition of the Maslov index.

Let

$$
\begin{aligned}
W: I=[0,1] & \rightarrow \mathcal{U}_{\mathcal{F}}\left(H_{J}\right) \\
t & \mapsto W(t)
\end{aligned}
$$

be a continuous path in $\mathcal{U}_{\mathcal{F}}\left(H_{J}\right)$. Since for Fredholm operators 0 is an isolated eigenvalue with finite multiplicity, we have the following property: 
Lemma 2.4. There exists a partition $0=t_{0}<t_{1}<\cdots<t_{N}=1$ of the interval $I$ and positive numbers $\varepsilon_{j}(j=1, \ldots, N)$ with $0<\varepsilon_{j}<\pi$ such that for each $t$ in $\left[t_{j-1}, t_{j}\right]$ the operator $W(t)-e^{\sqrt{-1}\left(\pi+\varepsilon_{j}\right)}$ is invertible, and also for each $t$ in $\left[t_{j-1}, t_{j}\right]$ the operator $W(t)$ has only a finite number of eigenvalues in the arc $\left\{e^{\sqrt{-1}(\pi+\theta)}|| \theta \mid \leq \varepsilon_{j}\right\}$.

We now define an 'index' $\mathbf{M}(\{W(t)\})$ of the curve $\{W(t)\}_{t \in I}$.

DeFinition 2.5. We set

$$
\mathbf{M}(\{W(t)\}):=\sum_{j=1}^{N}\left(k\left(t_{j}, \varepsilon_{j}\right)-k\left(t_{j-1}, \varepsilon_{j}\right)\right)
$$

with

$$
k\left(t, \epsilon_{j}\right):=\sum_{0 \leq \theta \leq \varepsilon_{j}} \operatorname{dim} \operatorname{Ker}\left(W(t)-e^{\sqrt{-1}(\pi+\theta)}\right)
$$

for $t_{j-1} \leq t \leq t_{j}$

As for Phillips's definition of the spectral flow (see $[\mathrm{Ph}]$ ) we see that the definition of the 'index' does not depend on the choice of the partition $0=t_{0}<t_{1}<$ $\cdots<t_{N}=1$ of the interval and the positive numbers $\left\{\varepsilon_{j}\right\}_{j=1}^{N}$ satisfying the above Lemma 2.4 .

This 'index' has the following properties:

(a) Additive under catenation of the paths,

(b) only a homotopy invariant of curves in $\mathcal{U}_{\mathcal{F}}\left(H_{J}\right)$ with fixed endpoints and distinguishes the homotopy class.

By making use of this 'index' we obtain now a functional analytic definition of the Maslov index for continuous paths in $\mathcal{F} \mathcal{L}_{\lambda}(H)$. Let $\mu: I \rightarrow \mathcal{F} \mathcal{L}_{\lambda}(H)$ be a continuous path in $\mathcal{F} \mathcal{L}_{\lambda}(H)$ (so that $\mathcal{S}_{\lambda} \circ \mu$ is a continuous path in $\mathcal{U}_{\mathcal{F}}\left(H_{J}\right)$ ).

Definition 2.6. We define the Maslov index of the curve $\{\mu(t)\}$ with respect to $\lambda$ by

$$
\operatorname{Mas}(\{\mu(t)\}, \lambda):=\mathbf{M}\left(\left\{\mathcal{S}_{\lambda}(\mu(t))\right\}\right) .
$$

By Proposition 2.3, the Maslov index inherits the properties of the 'index'.

REMARK 2.7. Let $\lambda^{\prime}$ be another Lagrangian subspace such that $\lambda \cap \lambda^{\prime}$ is of finite codimension in $\lambda$ (hence also in $\lambda^{\prime}$ ). We say in this case that $\lambda$ and $\lambda^{\prime}$ almost coincide or are almost coincident. This is an equivalent relation in $\mathcal{L}(H)$ and we have $\mathcal{F} \mathcal{L}_{\lambda}(H)=\mathcal{F} \mathcal{L}_{\lambda^{\prime}}(H)$ for almost coincident pair $\lambda$ and $\lambda^{\prime}$, and the difference of the Maslov index

$$
\operatorname{Mas}(\{\mu(t)\}, \lambda)-\operatorname{Mas}\left(\{\mu(t)\}, \lambda^{\prime}\right)
$$


only depends on the four Lagrangian subspaces $\mu(0), \mu(1), \lambda$ and $\lambda^{\prime}$. We denoted this quantity by $\sigma\left(\mu(1), \mu(0) ; \lambda, \lambda^{\prime}\right)$, and called it Hörmander index in the infinite dimension as a corresponding quantity to that for the finite dimensional case ([BF2]). This index works as the transition function in describing the universal covering space of $\mathcal{F} \mathcal{L}_{\lambda}(H)$.

In fact, let $\widetilde{\mathcal{F} \mathcal{L}_{\lambda}}(H)$ be the universal covering space of $\mathcal{F} \mathcal{L}_{\lambda}(H)$. We regard this space as the space of homotopy classes of continuous curves with the common initial point $=\lambda^{\perp}$ together with its end point. For $\mu \in \mathcal{L}(H)$ with the property that $\operatorname{dim} \lambda /(\mu \cap \lambda)<+\infty$, let us denote $\mathcal{F} \mathcal{L}_{\mu}^{(0)}=\mathcal{F} \mathcal{L}_{\mu}(H) \backslash \mathfrak{M}_{\mu}(H)=\mathcal{F} \mathcal{L}_{\lambda}(H) \backslash \mathfrak{M}_{\mu}(H)$ and define a map $g_{\mu}: \mathcal{F} \mathcal{L}_{\mu}^{(0)} \times \mathbb{Z} \rightarrow \widetilde{\mathcal{F} \mathcal{L}_{\lambda}}(H)$ by

$$
\left.g_{\mu}(x, n)=\text { (homotopy class of a curve }\{\gamma(t)\}, \gamma(1)\right)
$$

where $\{\gamma(t)\}$ is such a curve that $\gamma(0)=\lambda^{\perp}, \gamma(1)=x$ and $\operatorname{Mas}(\{\gamma(t)\}, \mu)=n$. Then the map $g_{\mu}$ gives a homeomorphism between $\mathcal{F} \mathcal{L}_{\mu}^{(0)} \times \mathbb{Z}$ and the subspace $\pi^{-1}\left(\mathcal{F} \mathcal{L}_{\mu}^{(0)}\right)$, where $\pi: \widetilde{\mathcal{F} \mathcal{L}_{\lambda}}(H) \rightarrow \mathcal{F} \mathcal{L}_{\lambda}(H)$ is the projection map. Now we know that such subspaces $\mathcal{F} \mathcal{L}_{\mu}^{(0)}$ cover $\mathcal{F} \mathcal{L}_{\lambda}(H)$ and for $x \in \mathcal{F} \mathcal{L}_{\mu}^{(0)} \cap \mathcal{F} \mathcal{L}_{\nu}^{(0)}, g_{\nu}^{-1} \circ g_{\mu}(x, n)=$ $\left(x, \sigma\left(x, \lambda^{\perp} ; \mu, \nu\right)+m\right)$, and we have the additivity $\sigma\left(x, \lambda^{\perp} ; \mu, \nu\right)+\sigma\left(x, \lambda^{\perp} ; \nu, \theta\right)=$ $\sigma\left(x, \lambda^{\perp} ; \mu, \theta\right)$ just by the definition. These show that $\mathcal{F L}_{\mu}^{(0)} \cap \mathcal{F} \mathcal{L}_{\nu}^{(0)} \ni x \mapsto$ $\sigma\left(x, \lambda^{\perp} ; \mu, \nu\right)$ is the transition function for the universal covering space of $\mathcal{F} \mathcal{L}_{\lambda}(H)$.

REMARK 2.8. In the finite dimensional case, the Maslov index for continuous paths was first defined in the paper [Go1] by noting the extendibility of the Leray index for arbitrary pairs of points on the universal covering space of Lagrangian Grassmannian through the Kashiwara index and by making use of the relation between Leray index and Maslov index. Conversely, first we define Maslov index for arbitrary paths with respect to a Maslov cycle as we gave above, then we can define Leray index for arbitrary pairs of points on the universal covering of Lagrangian Grassmannian.

In the infinite dimensional case we can define the Maslov index for arbitrary paths with respect to a Maslov cycle as we did above. But we cannot define Kashiwara index for arbitrary triples of Lagrangian subspaces as in the finite dimensional case, although we have a symmetric bilinear form similar to the finite dimensional case. We can define it for mutually almost coincident triples, since then the symmetric bilinear form is of finite rank. Also we cannot define Leray index for arbitrary pairs of points on the universal covering space of Fredholm-LagrangianGrassmannian. It might be possible only for pairs of unitary operators $U, V$ having the property that $U-V$ is of trace class through the embedding $\mathcal{S}_{\lambda}$ (see [KL]).

For differentiable curves, there is another way of describing the 'index' locally, which is analogous to Robbin-Salamon [RS]. Let $\{W(t)\}$ be a $C^{1}$-path in $\mathcal{U}_{\mathcal{F}}\left(H_{J}\right)$ with the differentiability with respect to the operator norm. 
Definition 2.9. (a) A parameter $t^{*}$ with $0<t^{*}<1$ is called a crossing for the family $\{W(t)\}$, if $\operatorname{Ker}\left(W\left(t^{*}\right)+\right.$ Id $) \neq\{0\}$.

(b) We define the crossing form at a crossing $t^{*}$ as a symmetric bilinear form on $\operatorname{Ker}\left(W\left(t^{*}\right)+\right.$ Id) by

$$
\widetilde{Q}_{\mathfrak{M}}(x, y):=\left.\frac{d}{d t}\langle x, R(t) y\rangle\right|_{t=t^{*}} \quad \text { for } x, y \in \operatorname{Ker}\left(W\left(t^{*}\right)+\mathrm{Id}\right)
$$

where $\{R(t)\}$ is a family of bounded selfadjoint operators defined by the condition $W(t)=W\left(t^{*}\right) e^{\sqrt{-1} R(t)}, R\left(t^{*}\right)=0$.

(c) A crossing $t^{*}$ is called regular if the form $\widetilde{Q}_{\mathfrak{M}}$ is non-degenerate at $t^{*}$.

Proposition 2.10. Let $\{W(t)\}$ be a path in $\mathcal{U}_{\mathcal{F}}\left(H_{J}\right)$ of class $C^{1}$ and $0<$ $t^{*}<1$ a regular crossing. Then there exists a real $\delta>0$ such that

$$
\mathbf{M}\left(\{W(t)\}_{\left|t-t^{*}\right| \leq \delta}\right)=\operatorname{sign} \widetilde{Q}_{\mathfrak{M}} .
$$

Proof. Suppose

$$
\operatorname{dim}_{\mathbb{C}} \operatorname{Ker}\left(W\left(t^{*}\right)-e^{\sqrt{-1} \pi}\right)=k>0
$$

then, since the eigenvalue -1 of $W\left(t^{*}\right)$ is isolated, there exists a positive number $\varepsilon>0$ such that for $0<|\theta| \leq \varepsilon$ the operator $W\left(t^{*}\right)-e^{\sqrt{-1}(\pi+\theta)}$ is invertible. Then we can choose a positive number $\delta_{0}>0$ such that for $\left|t-t^{*}\right| \leq \delta_{0}$ the operator $W(t)-e^{\sqrt{-1}(\pi \pm \varepsilon)}$ is invertible, and that

$$
\sum_{|\theta| \leq \varepsilon} \operatorname{dim} \operatorname{Ker}\left(W(t)-e^{\sqrt{-1}(\pi+\theta)}\right)=k
$$

for $\left|t-t^{*}\right| \leq \delta_{0}$. If the signature of $\widetilde{Q}_{\mathfrak{M}}$ is $(p, q)$, then by the regularity assumption at the crossing $t^{*}$, there exist eigenvalues of $\left.\dot{R}\left(t^{*}\right)\right|_{\operatorname{Ker}\left(W\left(t^{*}\right)+\mathrm{Id}\right)}$ such that

$$
0<\lambda_{1} \leq \cdots \leq \lambda_{p}, \quad 0>\mu_{1} \geq \cdots \geq \mu_{q} .
$$

By Kato's theorem (Theorem VIII 2.6 in [Ka]), there exists a $\delta>0$ (with $\delta<\delta_{0}$ ) such that for $t^{*} \leq t \leq t^{*}+\delta$, eigenvalues $\left\{\lambda_{i}(t)\right\}_{i=1}^{p}$ and $\left\{\mu_{i}(t)\right\}_{i=1}^{q}$ of $W(t)+\mathrm{Id}$ satisfy the following asymptotic property:

$$
\begin{aligned}
& \lambda_{i}(t)=\exp \left(\sqrt{-1}\left(\pi+\lambda_{i}\left(t-t^{*}\right)+o\left(\left|t-t^{*}\right|\right)\right)\right) \quad(i=1, \ldots, p) \\
& \mu_{j}(t)=\exp \left(\sqrt{-1}\left(\pi+\mu_{j}\left(t-t^{*}\right)+o\left(\left|t-t^{*}\right|\right)\right)\right) \quad(j=1, \ldots, q) .
\end{aligned}
$$


Therefore we have

$$
\begin{aligned}
& \sum_{0 \leq \theta \leq \varepsilon} \operatorname{dim} \operatorname{Ker}\left(W(t)-e^{\sqrt{-1}(\pi+\theta)}\right)=p, \\
& \sum_{-\varepsilon \leq \theta<0} \operatorname{dim} \operatorname{Ker}\left(W(t)-e^{\sqrt{-1}(\pi+\theta)}\right)=q
\end{aligned}
$$

for $t^{*}<t \leq t^{*}+\delta$. For $t^{*}-\delta \leq t<t^{*}, p$ and $q$ are exchanged.

Hence

$$
\begin{aligned}
\mathbf{M}\left(\{W(t)\}_{\left|t-t^{*}\right| \leq \delta}\right) & =k\left(t^{*}+\delta, \varepsilon\right)-k\left(t^{*}-\delta, \varepsilon\right) \\
& =p-q=\operatorname{sign} \widetilde{Q}_{\mathfrak{M}} .
\end{aligned}
$$

REMARK 2.11. (a) Kato's theorem ([Ka]) is proved for analytic curves, but this is also true for $C^{1}$-curves.

(b) For crossing $t^{*}=0$ or 1 , we only consider the one-sided differentiation in the definition of the crossing form. In these cases we have

$$
\begin{aligned}
\mathbf{M}\left(\{W(t)\}_{0 \leq t \leq \delta}\right) & =-q \\
\mathbf{M}\left(\{W(t)\}_{1-\delta \leq t \leq 1}\right) & =p^{\prime}
\end{aligned}
$$

where the signature of $\widetilde{Q}_{\mathfrak{M}}$ at $t^{*}=0$ is $(p, q)$ and at $t^{*}=1\left(p^{\prime}, q^{\prime}\right)$.

Corollary 2.12. Let $\mu: I \rightarrow \mathcal{F} \mathcal{L}_{\lambda}(H)$ be a $C^{1}$-class path (so that $\mathcal{S}_{\lambda} \circ \mu(t)$ is a path in $\mathcal{U}_{\mathcal{F}}\left(H_{J}\right)$ also of class $\left.C^{1}\right)$. Let $0<t^{*}<1$ be a regular crossing of the curve $\left\{\mathcal{S}_{\lambda} \circ \mu(t)\right\}$. Then there exists a $\delta>0$ such that

$$
\operatorname{Mas}\left(\{\mu(t)\}_{\left|t-t^{*}\right| \leq \delta}, \lambda\right)=\operatorname{sign} \widetilde{Q}_{\mathfrak{M}}
$$

where $\widetilde{Q}_{\mathfrak{M}}$ denotes the crossing form of $\left\{\mathcal{S}_{\lambda} \circ \mu(t)\right\}$ at the time $t=t^{*}$.

There is another differential description of the Maslov index which will turn out to be more suitable for proving the spectral flow formula. It is based on a representation of $\mu$ as the graph of a suitable bounded operator. Let $\mu: I \rightarrow$ $\mathcal{F} \mathcal{L}_{\lambda}(H)$ be a path in $\mathcal{F} \mathcal{L}_{\lambda}(H)$ of class $C^{1}$ and let $0<t^{*}<1$ be a crossing of the curve $\left\{\mathcal{S}_{\lambda} \circ \mu(t)\right\}$, i.e., $\mu\left(t^{*}\right) \cap \lambda \neq\{0\}$. Put $\mu=\mu\left(t^{*}\right)$, then $\mu(t)$ is transversal to $\mu^{\perp}$ for $\left|t-t^{*}\right| \ll 1$ and in this neighborhood of $t^{*}$, each $\mu(t)$ can be written as the graph of the bounded operator $\varphi(t): \mu \rightarrow \mu^{\perp}$. Note that the curve $\{\varphi(t)\}$ is also of class $C^{1}$. We consider the bilinear form

$$
Q_{\mathfrak{M}}(x, y):=\left.\frac{d}{d t} \omega(x, \varphi(t) y)\right|_{t=t^{*}} \quad \text { for } \quad x, y \in \mu\left(t^{*}\right) \cap \lambda
$$


It is a symmetric bilinear form and we have the following proposition.

Proposition 2.13. If $\mu\left(t^{*}\right) \cap \lambda \neq\{0\}$, then $\operatorname{sign} Q_{\mathfrak{M}}=\operatorname{sign} \widetilde{Q}_{\mathfrak{M}}$.

Proof. For $\left|t-t^{*}\right| \ll 1, \mu(t)$ is represented in two ways;

$$
\mu(t)=U(t)\left(\lambda^{\perp}\right)=\text { graph of } \varphi(t),
$$

where $U(t)$ is a unitary operator $\in \mathcal{U}_{\lambda}\left(H_{J}\right)$ and $\varphi: \mu \rightarrow \mu^{\perp}, \mu=\mu\left(t^{*}\right), \varphi\left(t^{*}\right)=0$.

We write operators $U(t)$ and $W(t)=U(t) \circ \theta_{\lambda}(U(t))$ in the following form;

$$
U(t)=U\left(t^{*}\right) e^{\sqrt{-1} A(t)}, \quad W(t)=W\left(t^{*}\right) e^{\sqrt{-1} R(t)},
$$

where $A(t)$ and $R(t)$ are selfadjoint operators with $A\left(t^{*}\right)=0$ and $R\left(t^{*}\right)=0$.

Now put $A(t)=X(t)+\sqrt{-1} Y(t)$ with $X={ }^{t} X, Y=-{ }^{t} Y$, then we obtain relations among differentials of $A(t), R(t)$ and $\varphi(t)$ at $t=t^{*}$ as follows;

$$
\begin{aligned}
\theta_{\lambda}\left(U\left(t^{*}\right)\right) \dot{R}\left(t^{*}\right) & =2 \dot{X}\left(t^{*}\right) \circ \theta_{\lambda}\left(U\left(t^{*}\right)\right) \\
U\left(t^{*}\right)^{-1}\left(-J \dot{\varphi}\left(t^{*}\right)\right) & =\dot{X}\left(t^{*}\right) U\left(t^{*}\right)^{-1} \text { on } \mu \cap \lambda .
\end{aligned}
$$

Therefore $\dot{R}\left(t^{*}\right)$ and $-J \dot{\varphi}\left(t^{*}\right)$ have the same signature on $\mu \cap \lambda$. By recalling that signatures of $R\left(t^{*}\right)$ and $-J \dot{\varphi}\left(t^{*}\right)$ are $\operatorname{sign} \widetilde{Q}_{\mathfrak{M}}$ and $\operatorname{sign} Q_{\mathfrak{M}}$ respectively, we complete the proof.

2.3. Maslov index for the curve of Fredholm pairs of Lagrangian subspaces. In the following we will denote the direct sum of the symplectic Hilbert space $(H, \omega)$ and $(H,-\omega)$ with the notation $\mathbb{H}=H \boxplus H \equiv H_{\omega} \oplus H_{-\omega}$. $\mathbb{H}$ is a symplectic Hilbert space with the symplectic form $\Omega(x \boxplus y, u \boxplus v)=\omega(x, u)$ $-\omega(y, v)$, and the corresponding almost complex structure $\mathbb{J}=J \oplus-J$, so that we have $\mathbb{H}_{\mathbb{J}}=H_{J} \oplus H_{-J}$.

Let $\left\{\left(\mu_{t}, \lambda_{t}\right)\right\}_{t \in I}$ be a continuous family of Fredholm pairs of Lagrangian subspaces, then $\left\{\mu_{t} \boxplus \lambda_{t}\right\}$ is a curve in $\mathcal{F} \mathcal{L}_{\Delta}(H \boxplus H)$, where $\Delta$ is the diagonal of $H \boxplus H$. Of course it is natural to define the Maslov index of the curve of Fredholm pairs $\left\{\left(\mu_{t}, \lambda_{t}\right)\right\}$ to be $\operatorname{Mas}\left(\left\{\mu_{t} \boxplus \lambda_{t}\right\}, \Delta\right)$.

Proposition 2.14. Let $\left\{\mu_{t}\right\}$ be a continuous curve in $\mathcal{F} \mathcal{L}_{\lambda}\left(H_{\omega}\right)$, then

$$
\operatorname{Mas}\left(\left\{\mu_{t}\right\}, \lambda\right)=\operatorname{Mas}\left(\left\{\mu_{t} \boxplus \lambda\right\}, \Delta\right) .
$$

REMARK 2.15. For loops the property may be well-known. For arbitrary continuous paths, in the finite dimensional case this may be proved by making use of relations with Leray index as we noted in Remark 2.8, but in the infinite dimensional case we have no such relations and we need a proof which is valid not only for loops but also for any continuous paths. 
If we identify $\mathbb{H}_{\mathbb{J}}=\Delta+\Delta^{\perp}=\Delta+\mathbb{J}(\Delta) \cong \Delta \otimes \mathbb{C}$, then $\tau_{\Delta}(a \boxplus b)=b \boxplus a$. Let us decompose $H$ as $H=\lambda \oplus \lambda^{\perp}$ and let $\varphi: \Delta \rightarrow \Delta^{\perp}$ be

$$
\varphi((x, y) \boxplus(x, y))=(-x, y) \boxplus(x,-y)
$$

where we express elements in $\Delta$ by $(x, y) \boxplus(x, y), x+y \in \lambda+\lambda^{\perp}=H$. Then we have

$$
\operatorname{graph} \varphi=\lambda^{\perp} \boxplus \lambda \text {. }
$$

Let $A=\mathbb{J} \circ \varphi: \Delta \rightarrow \Delta$ and $V: \mathbb{H}_{\mathbb{J}} \rightarrow \mathbb{H}_{\mathbb{J}}$ by

$$
V=\frac{-\sqrt{-1}}{\sqrt{2}}-\frac{A \otimes \mathrm{Id}}{\sqrt{2}}
$$

where we regard $A=A \otimes \mathrm{Id}$ is complexified according to the identification $\mathbb{H}_{J} \cong$ $\Delta \otimes \mathbb{C}$. Then we have

$$
\sqrt{-1}(A \otimes \mathrm{Id})((a, b) \boxplus(c, d))=(c,-d) \boxplus(-a, b)
$$

for $(a, b) \boxplus(c, d) \in H_{J} \boxplus H_{J}=\left(\lambda+\lambda^{\perp}\right) \boxplus\left(\lambda+\lambda^{\perp}\right)$ and

$$
V\left(\Delta^{\perp}\right)=\lambda^{\perp} \boxplus \lambda
$$

Now we define maps $\mathbf{a}_{\lambda}, \mathbf{b}_{\lambda}$ and $P_{\lambda}$ as follows:

$$
\begin{aligned}
\mathbf{a}_{\lambda}: \mathcal{U}_{\lambda}\left(H_{J}\right) & \longrightarrow \mathcal{U}_{\Delta}\left(\mathbb{H}_{\mathbb{J}}\right) \\
U & \mapsto \tilde{U} \circ V
\end{aligned}
$$

where $\widetilde{U}=U \oplus \operatorname{Id}: H_{J} \oplus H_{-J} \longrightarrow H_{J} \oplus H_{-J}$,

$$
\begin{aligned}
\mathbf{b}_{\lambda}: \mathcal{U}_{\mathcal{F}}\left(H_{J}\right) & \longrightarrow \quad \mathcal{U}_{\mathcal{F}}\left(\mathbb{H}_{\mathrm{J}}\right) \\
W & \mapsto \sqrt{-1} \cdot W \circ(A \otimes \mathrm{Id}),
\end{aligned}
$$

and

$$
\begin{aligned}
P_{\lambda}: \mathcal{F} \mathcal{L}_{\lambda}(H) & \rightarrow \mathcal{F} \mathcal{L}_{\Delta}(\mathbb{H}) \\
\mu & \mapsto \mu \boxplus \lambda .
\end{aligned}
$$

LemMA 2.16. The following diagram is commutative.

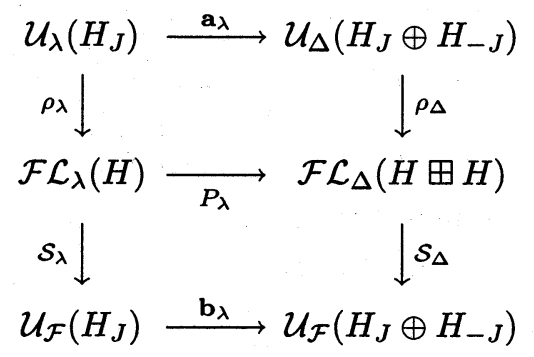


Proof. It will be enough to prove $\mathcal{S}_{\Delta} \circ P_{\lambda}=\mathbf{b}_{\lambda} \circ \mathcal{S}_{\lambda}$. Since $\theta_{\Delta}(V)=V$, $V^{2}=\sqrt{-1} \cdot A \otimes \operatorname{Id}$ and $\theta_{\Delta}(\widetilde{U})=\operatorname{Id} \boxplus U^{*}$ we have

$$
\begin{aligned}
\mathcal{S}_{\Delta} \circ \rho_{\Delta}\left(\mathbf{a}_{\lambda}(U)\right) & =\tilde{U} \circ V \circ \tau_{\Delta} \circ(\tilde{U} \circ V)^{*} \circ \tau_{\Delta} \\
& =\tilde{U} \circ \sqrt{-1}(A \otimes I \mathrm{Id}) \circ \theta_{\Delta}(\tilde{U}) \\
& =U \widetilde{U \theta_{\lambda}(U)} \circ \sqrt{-1}(A \otimes \mathrm{Id}),
\end{aligned}
$$

which prove the commutativity of the diagram.

Proof of Proposition 2.14. From the above lemma we can show that if $E$ is an eigenvalue of $\mathcal{S}_{\Delta}\left(\rho_{\Delta}\left(\mathbf{a}_{\lambda}(U)\right)\right)$, then $-E^{2}$ is an eigenvalue of $\mathcal{S}_{\lambda} \circ \rho_{\lambda}(U)$. Conversely if $l=e^{\sqrt{-1} \sigma}$ is an eigenvalue of $\mathcal{S}_{\lambda} \circ \rho_{\lambda}(U)$, then only one of $\pm e^{\sqrt{-1}(\pi+\sigma)}$ is close to -1 . So if we have a continuous curve $\left\{\mu_{t}\right\} \subset \mathcal{F} \mathcal{L}_{\lambda}(H)$, then the numbers of eigenvalues of $\left\{\mathcal{S}_{\lambda}\left(\mu_{t}\right)\right\}$ and $\left\{\mathcal{S}_{\Delta}\left(\mu_{t} \boxplus \lambda\right)\right\}$ across $e^{\sqrt{-1} \pi}$ coincide in both directions. This proves the proposition.

Also we can prove the following property in a similar way as above:

Proposition 2.17. $\operatorname{Mas}\left(\left\{\mu_{t} \boxplus \lambda_{t}\right\}, \Delta\right)=-\operatorname{Mas}\left(\left\{\lambda_{t} \boxplus \mu_{t}\right\}, \Delta\right)$.

\section{Spectral flow formula}

3.1. Symmetric operators and boundary value space. Let $H$ be a real separable Hilbert space and $A$ a densely defined closed symmetric operator with the domain $D_{m}$. Let $A^{*}$ denote its adjoint with the domain $D_{M}$.

Let $\boldsymbol{\beta}$ be the factor space of $D_{M}$ by $D_{m}, \boldsymbol{\beta}=D_{M} / D_{m}$, and let $\gamma: D_{M} \rightarrow \boldsymbol{\beta}$ $(\gamma(x)=[x])$ be the projection map. The space $\boldsymbol{\beta}$ becomes a symplectic Hilbert space with the inner product induced by the graph norm

$$
\langle x, y\rangle_{G}:=\langle x, y\rangle+\left\langle A^{*} x, A^{*} y\right\rangle
$$

on $D_{M}$ and the symplectic form given by 'Green's form'

$$
\omega([x],[y]):=\left\langle A^{*} x, y\right\rangle-\left\langle x, A^{*} y\right\rangle \text { for } x, y \in \boldsymbol{\beta} .
$$

For $D_{m} \subset D \subset D_{M}$, we denote

$$
A_{D}:=\left.A^{*}\right|_{D}
$$

and the following relations are fundamental for our theory:

$A_{D}$ is closed $\Leftrightarrow \gamma(D)$ is closed

$A_{D}$ is self-adjoint $\Leftrightarrow \gamma(D)$ is Lagrangian

$A_{D}$ has compact resolvent $\Leftrightarrow D \hookrightarrow H$ is compact in graph norm. 
(See [DS], Chapter XII.)

Definition 3.1. We call $\gamma\left(\operatorname{Ker} A^{*}\right)$ the Cauchy data space.

In fact if $A$ is an elliptic differential operator, then $\boldsymbol{\beta}$ can be embedded to the distribution space on the boundary manifold and $\gamma\left(\operatorname{Ker} A^{*}\right)$ is realized as the boundary values of $L_{2}$-solutions.

Now we make two assumptions.

Assumption (A). We assume that $A$ admits at least one self-adjoint Fredholm extension $A_{D}$ with the domain of definition $D \subset D_{M}$ and $A_{D}$ has the compact resolvent.

Then it follows ([BF1], Proposition 3.5) that $\gamma\left(\operatorname{Ker}\left(A^{*}\right)\right)$ is a Lagrangian subspace of $\boldsymbol{\beta}$ and $\left(\gamma\left(\operatorname{Ker} A^{*}\right), \gamma(D)\right)$ is a Fredholm pair.

Assumption (B). We assume that there exists a continuous curve $\left\{C_{t}\right\}$ in the space of bounded self-adjoint operators on $H$ and that the operators $A^{*}+C_{t}-s$ for small $s$ satisfy the "abstract unique continuation property";

$\operatorname{Ker}\left(A^{*}+C_{t}-s\right) \cap D_{m}=\{0\} \quad$ for $|s| \ll 1$ in a neighborhood of each $t$.

It follows (see [BF1], Theorem 3.8) that $\left\{\gamma\left(\operatorname{Ker}\left(A^{*}+C_{t}\right)\right)\right\}$ is a continuous curve in $\mathcal{F} \mathcal{L}_{\gamma(D)}(\boldsymbol{\beta})$.

3.2. Definition of the spectral flow. Let $H$ be a real separable Hilbert space and let $\widehat{\mathcal{F}}$ denote the space of bounded self-adjoint Fredholm operators on $H$. Following $[\mathrm{Ph}]$ we recall the definition of the spectral flow for continuous paths in $\widehat{\mathcal{F}}$.

Let $\left\{A_{t}\right\}_{t \in[0,1]}$ be a continuous path in $\widehat{\mathcal{F}}$.

Definition 3.2. We define the spectral flow by

$$
\mathbf{S f}\left(\left\{A_{t}\right\}\right):=\sum_{j=1}^{N}\left(k\left(t_{j}, \varepsilon_{j}\right)-k\left(t_{j-1}, \varepsilon_{j}\right)\right)
$$

where $\left\{t_{j}\right\}_{j=0}^{N}$, a partition of the interval $I$, and positive numbers $\varepsilon_{j}>0(j=$ $1, \ldots, N)$ have the following properties: the operator $A_{t}-\varepsilon_{j}$ is invertible for $t \in$ $\left[t_{j-1}, t_{j}\right]$ and the operator $A_{t}$ has only a finite number of eigenvalues in the interval $\left[-\varepsilon_{j}, \varepsilon_{j}\right]$ for each $t \in\left[t_{j-1}, t_{j}\right]$. Here we denote

$$
k\left(t, \varepsilon_{j}\right):=\sum_{0 \leq \theta<\varepsilon_{j}} \operatorname{dim} \operatorname{Ker}\left(A_{t}-\theta\right) \text { for } t_{j-1} \leq t \leq t_{j} .
$$


Similarly to the case of the Maslov index, the spectral flow is well defined for homotopy class of curves with fixed endpoints and it distinguishes the homotopy class of curves in $\widehat{\mathcal{F}}$ and is additive under catenation of curves. Especially, if the curve is in the trivial component $\widehat{\mathcal{F}}_{ \pm}$of $\widehat{\mathcal{F}}$, then it depends only on the end points and equals the difference $\sum_{\lambda<0} \operatorname{dim} \operatorname{Ker}\left(A_{0}-\lambda\right)-\sum_{\lambda<0} \operatorname{dim} \operatorname{Ker}\left(A_{1}-\lambda\right)$, in case $\left\{A_{t}\right\} \subset \widehat{\mathcal{F}}_{+}$and we have a similar formula for $\left\{A_{t}\right\} \subset \widehat{\mathcal{F}}_{-}$.

For $C^{1}$-class curves, there is another description of the spectral flow locally. On $\operatorname{Ker} A_{t^{*}}\left(0<t^{*}<1\right)$, we consider the well-defined symmetric bilinear form (crossing form) which is similar to $\S 2.2$ :

$$
Q_{\mathbf{S f}}(x, y):=\left.\frac{d}{d t}\left\langle x, A_{t}(y)\right\rangle\right|_{t=t^{*}} \quad \text { for } x, y \in \operatorname{Ker} A_{t^{*}}
$$

We have an analogous proposition to Proposition 2.10 .

Proposition 3.3. If the quadratic form $Q_{\mathbf{S f}}$ is non-degenerate on $\operatorname{Ker} A_{t^{*}}$, then for sufficiently small $\delta>0$, we have

$$
\mathbf{S f}\left(\left\{A_{t}\right\}_{\left|t-t^{*}\right| \leq \delta}\right)=\operatorname{sign}\left(\left.Q_{\mathbf{S f}}\right|_{\operatorname{Ker} A_{t^{*}}}\right)
$$

Proof. This is proved in a quite similar way as the proof of Proposition 2.10 .

Next we proceed to families of unbounded Fredholm self-adjoint operators $A_{t}=A_{D}+C_{t}$, as discussed in the preceding paragraph.

We assume the continuity of the family $\left\{C_{t}\right\}$ in $\mathcal{B}(H)$. First we apply the transformation $A \mapsto \mathcal{R}(A):=A{\sqrt{\mathrm{Id}+A^{2}}}^{-1}$ from the space $C \widehat{\mathcal{F}}$ of selfadjoint Fredholm operators to $\widehat{\mathcal{F}}$. The continuity of maps $t \mapsto \mathcal{R}\left(A_{D}+C_{t}\right)$ was proved in [BF1] (see also [CP]).

Definition 3.4. We define the spectral flow for $\left\{A_{D}+C_{t}\right\}_{t \in I}$ as

$$
\mathbf{S f}\left(\left\{A_{D}+C_{t}\right\}\right):=\mathbf{S f}\left(\left\{\mathcal{R}\left(A_{D}+C_{t}\right)\right\}\right)
$$

If the family $\left\{C_{t}\right\}$ is $C^{1}$-class in $\mathcal{B}(H)$, then we can define the crossing form as follows;

$$
Q_{0}(x, y):=\left.\frac{d}{d t}\left\langle x, C_{t}(y)\right\rangle\right|_{t=t^{*}} \quad \text { for } x, y \in \operatorname{Ker}\left(A^{*}+C_{t^{*}}\right)
$$


By the Proposition 2.10 in [CP], we know that $\mathcal{R}\left(A_{D}+C_{t}\right)$ is a $C^{1}$-class curve in $\widehat{\mathcal{F}}$ and the derivative is given by the formula

$$
\begin{aligned}
& \frac{d}{d t}\left(\mathcal{R}\left(A_{D}+C_{t}\right)\right) \\
& =\frac{1}{\pi} \int_{0}^{\infty} s^{-1 / 2}\left\{\left(1+\left(A_{D}+C_{t}\right)^{2}+s\right)^{-1}(1+s) \frac{d}{d t}\left(C_{t}\right)\left(1+\left(A_{D}+C_{t}\right)^{2}+s\right)^{-1}\right. \\
& \left.-\left(A_{D}+C_{t}\right)\left(1+\left(A_{D}+C_{t}\right)^{2}+s\right)^{-1} \frac{d}{d t}\left(C_{t}\right)\left(A_{D}+C_{t}\right)\left(1+\left(A_{D}+C_{t}\right)^{2}+s\right)^{-1}\right\} d s
\end{aligned}
$$
we have

In particular for $x \in \operatorname{Ker}\left(A_{D}+C_{t}\right)$ we have $\frac{d}{d t}\left(\mathcal{R}\left(A_{D}+C_{t}\right)\right)(x)=\frac{d}{d t} C_{t}(x)$, so

Proposition 3.5. $Q_{0}(x, y)=Q_{\mathbf{S f}}(x, y)$ at crossing $t=t^{*}$ for any $x, y \in$ $\operatorname{Ker}\left(A_{D}+C_{t^{*}}\right)$.

3.3. Local spectral flow formula. It would be interesting for us to derive a local identification of the spectral flow with the Maslov index for curves of $C^{1}$ class. We inherit the notations and the assumptions from $§ 3.1$. We have first the following

Proposition 3.6. For any $t^{*} \in[0,1]$ and $x, y \in \operatorname{Ker}\left(A^{*}+C_{t}\right)$,

$$
Q_{0}(x, y)=Q_{\mathfrak{M}}(\gamma(x), \gamma(y))
$$

Proof. When $S_{t^{*}}=\operatorname{Ker}\left(A^{*}+C_{t}\right) \neq 0$, write $\gamma\left(\operatorname{Ker}\left(A^{*}+C_{t}\right)\right)=\lambda_{t}=$ graph $\varphi_{t}$, where $\varphi_{t}: \lambda_{t^{*}} \rightarrow \lambda_{t^{*}}^{\perp}$ for $\left|t-t^{*}\right| \ll 1$. We can obtain the lift of $\varphi_{t}$ as follows: Fix a closed direct summand $\mu$ in $D_{M}$ as $D_{M}=S_{t^{*}} \oplus D_{m} \oplus \mu$, then we can define

$$
\begin{aligned}
f_{t} \oplus \psi_{t}: S_{t^{*}} & \rightarrow D_{m} \oplus \mu \\
x & \mapsto f_{t}(x) \oplus \psi_{t}(x)
\end{aligned}
$$

such that $S_{t}=\operatorname{graph} f_{t} \oplus \psi_{t}$. By the definition of the crossing form and the symplectic structure on $\beta$, we have

$$
\begin{aligned}
Q_{\mathfrak{M}}(\gamma(x), \gamma(y)) & =Q_{\mathfrak{M}}([x],[y])=\left.\frac{d}{d t} \omega\left([x], \varphi_{t}[y]\right)\right|_{t=t^{*}} \\
& =\left.\frac{d}{d t}\left\{\left\langle A^{*} x, \psi_{t}(y)\right\rangle-\left\langle x, A^{*}\left(\psi_{t}(y)\right)\right\rangle\right\}\right|_{t=t^{*}} .
\end{aligned}
$$

Since

$$
\left(A^{*}+C_{t}\right)\left(y+f_{t}(y)+\psi_{t}(y)\right)=0 \text { for all } y \in S_{t^{*}}
$$


by differentiating the above equation with respect to $t$ and evaluating at $t=t^{*}$, we obtain

$$
\dot{C}_{t^{*}}(y)+\left(A^{*}+C_{t^{*}}\right)\left(\dot{f}_{t^{*}}(y)+\dot{\psi}_{t^{*}}(y)\right)=0
$$

Therefore,

$$
A^{*}\left(\dot{\psi}_{t^{*}}(y)\right)=-\dot{C}_{t^{*}}(y)-\left(A^{*}+C_{t^{*}}\right)\left(\dot{f}_{t^{*}}(y)\right)-C_{t^{*}}\left(\dot{\psi}_{t^{*}}(y)\right)
$$

By substituting this result into $(*)$, we get

$$
(*)=\left\langle A^{*} x, \dot{\psi}_{t^{*}}(y)\right\rangle+\left\langle x, \dot{C}_{t^{*}}(y)+\left(A^{*}+C_{t^{*}}\right)\left(\dot{f}_{t^{*}}(y)\right)+C_{t^{*}}\left(\dot{\psi}_{t^{*}}(y)\right)\right\rangle .
$$

Since $\left(A^{*}+C_{t^{*}}\right) x=0$ and $\dot{f}_{t^{*}}(y) \in D_{m}$, we have

$$
\begin{aligned}
& \left\langle A^{*} x, \dot{\psi}_{t^{*}}(y)\right\rangle+\left\langle x, C_{t^{*}}\left(\dot{\psi}_{t^{*}}(y)\right)\right\rangle=\left\langle\left(A^{*}+C_{t^{*}}\right)(x), \dot{\psi}_{t^{*}}(y)\right\rangle=0 \\
& \left\langle x,\left(A^{*}+C_{t^{*}}\right)\left(\dot{f}_{t^{*}}(y)\right)\right\rangle=\left\langle x,\left(A+C_{t^{*}}\right)\left(\dot{f}_{t^{*}}(y)\right)\right\rangle \\
& =\left\langle\left(A^{*}+C_{t^{*}}\right)(x), \dot{f}_{t^{*}}(y)\right\rangle=0 \text {. }
\end{aligned}
$$

Therefore we obtain $(*)=\left\langle x, \dot{C}_{t^{*}}(y)\right\rangle$, which completes the proof.

Combining Proposition 3.3 with Proposition 3.6 and Corollary 2.12 yields the following local spectral formula.

TheOREM 3.7. If the quadratic form $Q_{\mathbf{S f}}$ is non-degenerate on $S_{t^{*}} \cap D$, then we have

$$
\mathbf{S f}\left(\left\{A_{D}+C_{t}\right\}_{\left|t-t^{*}\right| \leq \delta}\right)=\operatorname{Mas}\left(\left\{\lambda_{t}\right\}_{\left|t-t^{*}\right| \leq \delta}, \gamma(D)\right)
$$

for sufficient small $\delta>0$.

3.4. General spectral flow formula. Under assumptions made in $\S 3.1$ (Assumption (A) and Assumption (B)), we obtain a general spectral flow formula. This theorem was proved in [BF1], Theorem 5.1. Here we give a proof of the formula by emphasizing the local spectral flow formula (Theorem 3.7) in the preceding section.

Theorem 3.8. Let $\left\{A_{D}+C_{t}\right\}$ be a family satisfying Assumption (A) and (B). Then we have

$$
\mathbf{S f}\left(\left\{A_{D}+C_{t}\right\}\right)=\operatorname{Mas}\left(\left\{\gamma\left(S_{t}\right)\right\}, \gamma(D)\right)
$$

Proof. Recalling the additivity of the spectral flow and the Maslov index under catenation of two curves, it is sufficient to prove that

$$
\mathbf{S f}\left(\left\{A_{D}+C_{t}\right\}_{t_{1} \leq t \leq t_{2}}\right)=\operatorname{Mas}\left(\left\{\gamma\left(S_{t}\right)\right\}_{t_{1} \leq t \leq t_{2}}, \gamma(D)\right) \quad \text { for } 0<t_{2}-t_{1} \ll 1 .
$$


We deform the curve $\left\{A_{D}+C_{t}\right\}_{t_{1} \leq t \leq t_{2}}$ to $\left\{A_{D}+C_{t_{1}}+s\right\}_{0 \leq s \leq \varepsilon} \cup\left\{A_{D}+C_{t}+\right.$ $\varepsilon\}_{t_{1} \leq t \leq t_{2}} \cup\left\{A_{D}+C_{t_{2}}+\varepsilon-s\right\}_{0 \leq s \leq \varepsilon}$, provided that for $t_{1} \leq t \leq t_{2}$ and $0 \leq s \leq \varepsilon$, Assumption (B) is satisfied.

By the property of homotopy invariance, the spectral flow and the Maslov index are equal respectively for previous two curves.

Since $\operatorname{Ker}\left(A_{D}+C_{t}+\varepsilon\right)=0$ for $t_{1} \leq t \leq t_{2}$, the spectral flow and the Maslov index vanish for corresponding curve. By applying local spectral flow formula (Theorem 3.7) to the smooth curves $\left\{A_{D}+C_{t_{1}}+s\right\}_{0 \leq s \leq \varepsilon}$ and $\left\{A_{D}+C_{t_{2}}+\varepsilon-s\right\}_{0 \leq s \leq \varepsilon}$, we get the result of this theorem.

\section{Splitting formula for a spectral flow}

In this section we prove our main Theorem 4.12.

Let $\mathbb{E}$ be a real vector bundle on a closed manifold $M$ (we assume $M$ is connected), and let $A$ be an elliptic and selfadjoint first order differential operator acting on the vector bundle $\mathbb{E}$. The selfadjointness of the operator $A$ will be with respect to a suitably fixed $L_{2}$-inner product on the space of smooth sections $C^{\infty}(M, \mathbb{E})$. We will not describe the inner product on $\mathbb{E}$ explicitly. Let $\left\{C_{t}\right\}$ be a continuous family of selfadjoint bundle maps of $\mathbb{E}$, and we will regard them, using the same notation, as a continuous family of zeroth order operators on $L_{2}(M, \mathbb{E})$. We also denote by $H^{s}(M, \mathbb{E})$ a Sobolev space of sections of $\mathbb{E}$ of order $s$. Throughout this section we assume that the operators $A+C_{t}+s(|s| \ll 1)$ satisfy the unique continuation property for any hypersurface (Assumption (B)).

4.1. Splitting of manifolds and first order differential operators. Let $\Sigma$ be a hypersurface on $M$ and let $D_{\min }$ be a subspace in $H^{1}(M, \mathbb{E})$ consisting of sections which vanish on $\Sigma$. Then the operator $A$ defined on $D_{\min }$, denoted by $\mathcal{A}_{0}$, is a closed symmetric operator. We will denote the domain of the adjoint operator $\left(\mathcal{A}_{0}\right)^{*}$ by $D_{\max }$. Here in addition if we assume that the hypersurface separates the manifold $M$ into two parts $M_{ \pm}$with common boundary $\Sigma=\partial M_{ \pm}$, then $D_{\min }$ and $D_{\max }$ are also decomposed into two components corresponding to submanifolds $M_{ \pm}$. We denote them by $D_{\min }=D_{\min }^{-} \oplus D_{\min }^{+}, D_{\max }=D_{\max }^{-} \oplus D_{\max }^{+}$and $\mathcal{A}_{0}=\mathcal{A}_{0}^{-} \oplus \mathcal{A}_{0}^{+}$, and $D_{\text {max }}^{ \pm}$is the domain of the adjoint operator of $\mathcal{A}_{0}^{ \pm}$respectively. Although graph inner products on $D_{\max }$ by operator $A+C_{t}$ are not identical, the norms are all equivalent and the symplectic forms $\omega_{ \pm}$on $\beta^{ \pm}=D_{\max }^{ \pm} / D_{\min }^{ \pm}$do not depend on the parameter.

Notice that if $\Sigma$ is only orientable and does not separate $M$, that is, if $M \backslash \Sigma$ is connected, then $D_{\min }$ (and also $D_{\max }$ ) does not decompose in the above way, but $\boldsymbol{\beta}=D_{\max } / D_{\min }$ is a sum of two spaces $\boldsymbol{\beta}^{ \pm}$of boundary values taken from each side. However in this case the Cauchy data space $\gamma\left(\operatorname{Ker}\left(\mathcal{A}_{0}\right)^{*}\right)$ is not a sum of two Lagrangian subspaces in $\beta^{ \pm}$. 
Henceforth we assume that a hypersurface $\Sigma$ separates $M$ into two parts $M_{ \pm}$.

Proposition 4.1 ([Ho1]). $\quad \boldsymbol{\beta}^{ \pm}$is a subspace in $H^{-1 / 2}\left(\Sigma,\left.\mathbb{E}\right|_{\Sigma}\right)$, the Sobolev space of sections with values in the bundle $\mathbb{E}$ restricted to $\Sigma$, of order $-1 / 2$. Also we have $\boldsymbol{\beta}^{-} \cap \boldsymbol{\beta}^{+}=H^{1 / 2}\left(\Sigma,\left.\mathbb{E}\right|_{\Sigma}\right)$.

Let $\gamma_{ \pm}$be the map from $D_{\max }^{ \pm}$to $\boldsymbol{\beta}^{ \pm}$. The families $\left\{\Lambda_{t}^{ \pm}\right\}=\left\{\gamma_{ \pm}\left(\operatorname{Ker}\left(\left(\mathcal{A}_{0}^{ \pm}+\right.\right.\right.\right.$ $\left.\left.\left.\left.C_{t}\right)^{*}\right)\right)\right\}$ are continuous families of Lagrangian subspaces of $\boldsymbol{\beta}^{ \pm}$and according to the general spectral flow formula in $§ 3$ we have

THEOREM 4.2. From the ellipticity of the operator we have $\Lambda_{t}^{-} \oplus \Lambda_{t}^{+} \epsilon$ $\mathcal{F} \mathcal{L}_{\boldsymbol{\delta}}(\boldsymbol{\beta})$, and $\mathbf{S f}\left\{A+C_{t}\right\}=\operatorname{Mas}\left(\left\{\Lambda_{t}^{-} \oplus \Lambda_{t}^{+}\right\}, \boldsymbol{\delta}\right)$ where

$$
\boldsymbol{\delta}=\left\{\left.(\varphi, \varphi) \in \boldsymbol{\beta}^{-} \oplus \boldsymbol{\beta}^{+}\right|^{\exists} f \in H^{1}(M, \mathbb{E}), \gamma_{ \pm}\left(\left.f\right|_{M_{ \pm}}\right)=\varphi\right\}
$$

Let $\left\{L_{s, t}\right\}$ be a family of bounded operators on $L_{2}(M, \mathbb{E})$ defined as

$$
L_{s, t}(f)= \begin{cases}0 & \text { on } M_{-} \\ \left(C_{t}-C_{s}\right)(f) & \text { on } M_{+}\end{cases}
$$

It is easy to see that the two-parameter family $\left\{L_{s, t}\right\}$ is a continuous family of bounded selfadjoint operators on $L_{2}(M, \mathbb{E})$.

We define $\left\{\mathcal{A}_{s, t}\right\}$ a two-parameter family of operators on $H^{1}(M, \mathbb{E})$ defined as

$$
\mathcal{A}_{s, t}(f)=\left\{\begin{array}{lll}
\left(A+C_{s}\right)(f) & \text { on } & M_{-} \\
\left(A+C_{t}\right)(f) & \text { on } & M_{+}
\end{array}\right.
$$

then since $\mathcal{A}_{s, t}=A+C_{s}+L_{s, t}$ we have

Proposition 4.3. Each operator $\mathcal{A}_{s, t}$ satisfies the 'a priori' estimate

$$
\|u\|_{1} \leq C\left(\left\|\mathcal{A}_{s, t}(u)\right\|_{0}+\|u\|_{0}\right)
$$

with a uniform constant $C>0$.

Here we denote $\|u\|_{1}$ the first order Sobolev norm of $u \in H^{1}(M, \mathbb{E})$.

By this proposition we see that the direct sum $\Lambda_{s}^{-} \oplus \Lambda_{t}^{+}$of the Cauchy data spaces of the operator $\left(\mathcal{A}_{0}^{-}\right)^{*}+C_{s}$ and $\left(\mathcal{A}_{0}^{+}\right)^{*}+C_{t}$ give rise to a Fredholm pair in $\boldsymbol{\beta}^{-} \oplus \boldsymbol{\beta}^{+}$and the family $\left\{\Lambda_{s}^{-} \oplus \Lambda_{t}^{+}\right\}$is a continuous two-parameter family of Lagrangian subspaces in $\boldsymbol{\beta}^{-} \oplus \boldsymbol{\beta}^{+}$. Hence we have

Theorem 4.4. $\quad \mathbf{S f}\left\{A+C_{t}\right\}=\mathbf{S f}\left(\left\{\mathcal{A}_{s, 0}\right\}_{s \in I}\right)+\mathbf{S f}\left(\left\{\mathcal{A}_{1, t}\right\}_{t \in I}\right)$.

This formula can be seen as a splitting formula of the spectral flow for the family $\left\{A+C_{t}\right\}$. However each term in the right hand side is a spectral flow 
corresponding to the operator family $\left\{\mathcal{A}_{s, 0}\right\}_{s \in I}$ and $\left\{\mathcal{A}_{1, t}\right\}_{t \in I}$ on the whole manifold $M$. The purpose of this paper is to study when we can express these spectral flows in terms of spectral flows of families of operators restricted to the separated submanifolds $M_{ \pm}$. We are not sure whether such a kind of splitting formula holds for any family of selfadjoint elliptic operators. One difficulty for expressing each term as a spectral flow of a family defined on the component $M_{ \pm}$lies in the general spectral flow formula. Because there we have a formula for the spectral flow in terms of the Maslov index in the distribution space $\boldsymbol{\beta}$ and these two spaces of boundary values $\boldsymbol{\beta}^{ \pm}$taken from each side of the separating hypersurface $\Sigma$ are not identical even for the case of the operators of the product form in a neighborhood of $\Sigma$. So it will be natural to expect that if we have a reformulation of the spectral flow formula in terms of the $L_{2}$-space on the hypersurface $\Sigma$, then we will have such a splitting formula. We would like to make these situations clearer. So we recall in the next subsection a reduction formula for the Maslov index in the infinite dimension.

4.2. A reduction formula for the Maslov index. Let $\boldsymbol{\beta}$ and $L$ be two symplectic Hilbert spaces with the symplectic form $\omega_{\boldsymbol{\beta}}$ and $\omega_{L}$. We assume that each space $\boldsymbol{\beta}$ and $L$ decomposes as a direct sum of two Lagrangian subspaces in the following way:

$$
\boldsymbol{\beta}=\theta_{-} \oplus \theta_{+}
$$

and

$$
L=L_{-} \oplus L_{+}
$$

We also assume that there are injective maps

$$
\begin{aligned}
& \mathbf{i}_{-}: \theta_{-} \rightarrow L_{-} \\
& \mathbf{i}_{+}: L_{+} \rightarrow \theta_{+}
\end{aligned}
$$

such that $\omega_{L}\left(\mathbf{i}_{-}(x), y\right)=\omega_{\boldsymbol{\beta}}\left(x, \mathbf{i}_{+}(y)\right)$ for $x \in \theta_{-}$and $y \in L_{+}$, and moreover we assume that the images under these maps are dense.

Then we have

Theorem 4.5 ([BFO]). There is a natural continuous map

$$
\ell: \mathcal{F} \mathcal{L}_{\theta_{-}}(\boldsymbol{\beta}) \rightarrow \mathcal{F} \mathcal{L}_{L_{-}}(L)
$$

satisfying

$$
\operatorname{Mas}\left(\left\{\mu_{t}\right\}, \theta_{-}\right)=\operatorname{Mas}\left(\left\{\ell\left(\mu_{t}\right)\right\}, L_{-}\right)
$$


4.3. Splitting formula for a spectral flow. From this section on we assume that the operators $A+C_{s}$ appearing in the family are all of the product form near the hypersurface $\Sigma$, that is, the operator $A+C_{s}$ has the following form on a cylindrical neighborhood $U(\Sigma) \cong(-1,1) \times \Sigma$ of $\Sigma$ :

$$
A+C_{s}=\sigma\left(\frac{\partial}{\partial \tau}+B_{s}\right)
$$

where $\tau \in(-1,1), \sigma$ is a bundle map of $\left.\mathbb{E}\right|_{\Sigma}$ defined on $\Sigma, B_{s}$ is a selfadjoint elliptic operator on $\left.\mathbb{E}\right|_{\Sigma}, \pi^{*}\left(\left.\left.\mathbb{E}\right|_{\Sigma} \cong \mathbb{E}\right|_{U(\Sigma)}\right.$, and $\pi: U(\Sigma) \rightarrow \Sigma$ is defined through a fixed identification $(-1,1) \times \Sigma \cong U(\Sigma)$.

Let $\left\{\varphi_{k}\right\}_{k \in \mathbb{Z} \backslash\{0\}}$ be a complete orthonormal system of eigen-sections of the operator $B_{0}$, then we have a concrete characterization of the boundary value space $\boldsymbol{\beta}^{ \pm}$as follows.

Proposition 4.6 ([BF2]).

$$
\begin{aligned}
& \boldsymbol{\beta}^{+}={\overline{\left[\left\{\varphi_{k}\right\}\right.}}_{k<0}^{H^{1 / 2}(\Sigma)} \oplus{\overline{\left[\left\{\varphi_{k}\right\}\right.}}_{k>0}^{H^{-1 / 2}(\Sigma)}=\mathcal{B}_{-}^{+}+\mathcal{B}_{+}^{+}
\end{aligned}
$$

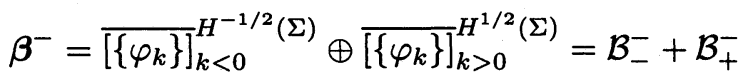

where we mean by $\mathcal{B}_{-}^{+}={\overline{\left[\left\{\varphi_{k}\right\}\right.}}_{k<0}^{H^{1 / 2}(\Sigma)}$ the completion of the space $\left[\left\{\varphi_{k}\right\}_{k<0}\right]$ spanned by $\left\{\varphi_{k}\right\}_{k<0}$ with respect to the Sobolev norm $H^{1 / 2}(\Sigma)=H^{1 / 2}\left(\Sigma,\left.\mathbb{E}\right|_{\Sigma}\right)$ and so on.

Let us consider the family of operators $\left\{\mathcal{A}_{s, 0}\right\}$

$$
\mathcal{A}_{s, 0}(f)=\left\{\begin{array}{lll}
\left(A+C_{s}\right)(f) & \text { on } & M_{-} \\
\left(A+C_{0}\right)(f) & \text { on } & M_{+}
\end{array}\right.
$$

for $f \in H^{1}(M, \mathbb{E})$. The spectral flow of this family is equal to $\operatorname{Mas}\left(\left\{\Lambda_{s}^{-} \oplus \Lambda_{0}^{+}\right\}, \delta\right)$ by our general spectral flow formula (Theorem 3.8).

Then let us take $\beta$ and $L$ in the Theorem 4.5 as

$$
\begin{aligned}
& \boldsymbol{\beta}=\boldsymbol{\beta}^{-} \oplus \boldsymbol{\beta}^{+} \\
& L=L_{2}\left(\Sigma,\left.\mathbb{E}\right|_{\Sigma}\right) \oplus L_{2}\left(-\Sigma,\left.\mathbb{E}\right|_{\Sigma}\right)=L_{2}\left(\Sigma,\left.\mathbb{E}\right|_{\Sigma}\right) \boxplus L_{2}\left(\Sigma,\left.\mathbb{E}\right|_{\Sigma}\right) .
\end{aligned}
$$

Here $L_{2}(-\Sigma)=L_{2}\left(-\Sigma,\left.\mathbb{E}\right|_{\Sigma}\right)$ means the symplectic Hilbert space with the opposite sign of symplectic structure of $L_{2}\left(\Sigma,\left.\mathbb{E}\right|_{\Sigma}\right)$, or the orientation of $\Sigma$ should be reversed.

We have decompositions of $\beta=\theta_{-} \oplus \theta_{+}$and $L=L_{-} \oplus L_{+}$as follows:

$$
\theta_{-}=\boldsymbol{\delta}=\left\{\left.(\varphi, \varphi) \in \boldsymbol{\beta}^{-} \oplus \boldsymbol{\beta}^{+}\right|^{\exists} f \in H^{1}(M, \mathbb{E}), \gamma_{ \pm}\left(\left.f\right|_{M_{ \pm}}\right)=\varphi\right\}
$$


$\theta_{+}=\left\{(x, 0,0, y) \in \mathcal{B}_{-}^{-}+\mathcal{B}_{+}^{-}+\mathcal{B}_{-}^{+}+\mathcal{B}_{+}^{+} \mid x \in{\overline{\left[\left\{\varphi_{k}\right\}\right.}}_{k<0}^{H^{-1 / 2}(\Sigma)}, y \in{\overline{\left[\left\{\varphi_{k}\right\}\right]}}_{k>0}^{H^{-1 / 2}(\Sigma)}\right\}$,

$L_{-}=\Delta=$ the diagonal of $L_{2}\left(\Sigma,\left.\mathbb{E}\right|_{\Sigma}\right) \boxplus L_{2}\left(\Sigma,\left.\mathbb{E}\right|_{\Sigma}\right)$,

$L_{+}=\left\{(u, 0,0, v) \mid u \in{\overline{\left[\left\{\varphi_{k}\right\}\right.}}_{k<0}^{L_{2}(\Sigma)}, v \in{\overline{\left[\left\{\varphi_{k}\right\}\right.}}_{k>0}^{L_{2}(\Sigma)}\right\}$.

We have obvious embeddings $\mathbf{i}_{-}: \theta_{-} \rightarrow L_{-}$and $\mathbf{i}_{+}: L_{+} \rightarrow \theta_{+}$in the space of distributions on $\Sigma$ satisfying the properties as required in Theorem 4.5. Then we have a spectral flow formula in the $L_{2}$ setting, since in this case the map $\ell$ means just taking the intersection in the distribution space on $\Sigma$ :

Proposition 4.7. $\operatorname{Sf}\left\{\mathcal{A}_{s, 0}\right\}=\operatorname{Mas}\left(\left\{\left(\Lambda_{s}^{-} \cap L_{2}(\Sigma)\right) \boxplus\left(\Lambda_{0}^{+} \cap L_{2}(\Sigma)\right)\right\}, \Delta\right)$.

Now from Proposition 2.14 we have

Proposition 4.8 .

$$
\begin{array}{r}
\operatorname{Mas}\left(\left\{\left(\Lambda_{s}^{-} \cap L_{2}(\Sigma)\right) \boxplus\left(\Lambda_{0}^{+} \cap L_{2}(\Sigma)\right)\right\}, \Delta\right) \\
=\operatorname{Mas}\left(\left\{\Lambda_{s}^{-} \cap L_{2}(\Sigma)\right\}, \Lambda_{0}^{+} \cap L_{2}(\Sigma)\right) .
\end{array}
$$

Next let us consider the family of operators $\left\{\mathcal{T}_{s}^{-}\right\}$on $M_{-}$with the domain $D_{0}$,

$$
D_{0}=\left\{\left.f \in H^{1}\left(M_{-},\left.\mathbb{E}\right|_{M_{-}}\right)\right|^{\exists} \varphi \in \Lambda_{0}^{+}, \gamma_{-}(f)=\varphi\right\}
$$

and

$$
\mathcal{T}_{s}^{-}(f)=\left(A+C_{s}\right)(f) \text { on } M_{-} .
$$

From the reduction theorem $4.5, \gamma_{-}\left(D_{0}\right)$ is a Lagrangian subspace in $\boldsymbol{\beta}^{-}$. Hence we have

Proposition 4.9. Each operator $\mathcal{T}_{s}^{-}$on $D_{0}$ is a selfadjoint operator. So this implies that

$$
\|u\|_{1} \leq c^{\prime}\left(\left\|\mathcal{T}_{s}^{-} u\right\|_{0}+\|u\|\right) \quad \text { for } u \in D_{0}
$$

with a uniform constant $c^{\prime}>0$. This inequality implies that we have well-defined spectral flow for this continuous family $\left\{\mathcal{T}_{s}^{-} \circ{\sqrt{1+\left(\mathcal{T}_{s}^{-}\right)^{2}}}^{-1}\right\}$.

Hence we have

Proposition 4.10.

$$
\begin{aligned}
\mathbf{S f}\left(\left\{\mathcal{T}_{s}^{-}\right\}\right) & =\operatorname{Mas}\left(\left\{\Lambda_{s}^{-}\right\}, \gamma_{-}\left(D_{0}\right)\right) \\
& =\operatorname{Mas}\left(\left\{\Lambda_{s}^{-} \cap L_{2}(\Sigma)\right\}, \Lambda_{0}^{+} \cap L_{2}(\Sigma)\right) \\
& =\operatorname{Sf}\left\{\mathcal{A}_{s, 0}\right\}
\end{aligned}
$$


Similarly when we define the family of operators $\left\{\mathcal{T}_{t}^{+}\right\}$on $M_{+}$with the domain $D_{1}$ given by

$$
D_{1}=\left\{\left.v \in H^{1}\left(M_{+},\left.\mathbb{E}\right|_{M_{+}}\right)\right|^{\exists} \varphi \in \Lambda_{1}^{-}, \gamma_{+}(v)=\varphi\right\}
$$

and

$$
\mathcal{T}_{t}^{+}(v)=\left(A+C_{t}\right) v \text { on } M_{+},
$$

then we have

Proposition 4.11.

$$
\begin{aligned}
\mathbf{S f}\left(\left\{\mathcal{T}_{t}^{+}\right\}\right) & =\operatorname{Mas}\left(\left\{\Lambda_{t}^{+}\right\}, \gamma_{+}\left(D_{1}\right)\right) \\
& =\operatorname{Mas}\left(\left\{\Lambda_{t}^{+} \cap L_{2}(\Sigma)\right\}, \Lambda_{1}^{-} \cap L_{2}(\Sigma)\right) \\
& =\operatorname{Sf}\left\{\mathcal{A}_{0, t}\right\} .
\end{aligned}
$$

Summing up the results above we have our main Theorem of a splitting formula for the spectral flow $\mathbf{S f}\left\{A+C_{t}\right\}$.

Theorem 4.12. $\operatorname{Sf}\left\{A+C_{t}\right\}=\operatorname{Sf}\left\{\mathcal{T}_{t}^{-}\right\}+\operatorname{Sf}\left\{\mathcal{T}_{t}^{+}\right\}$.

Remark 4.13. (a) We have proved Theorem 4.12 under the assumption of the product form structure for the operators near the hypersurface $\Sigma$. It is important that this assumption implies that the conditions to define the domains $D_{0}$ and $D_{1}$ become selfadjoint elliptic boundary conditions.

(b) If the operator is not of product form near the hypersurface $\Sigma$, but if an operator $A+C_{0}$ is invertible, then we can prove that the condition in (4.2) gives us an elliptic selfadjoint boundary condition.

Proof. The invertibility of the operator $A+C_{0}$ is equivalent to the transversality of the two Lagrangian subspaces $\delta$ and $\Lambda_{0}^{-} \oplus \Lambda_{0}^{+}$in $\boldsymbol{\beta}=\boldsymbol{\beta}^{-} \oplus \boldsymbol{\beta}^{+}$. Hence any $(z, 0) \in \boldsymbol{\beta}^{-} \oplus \boldsymbol{\beta}^{+}$can be written as $(z, 0)=(x, y)+(a, a) \in \Lambda_{0}^{-} \oplus \Lambda_{0}^{+}+\boldsymbol{\delta}$. So $a=-y \in H^{1 / 2}\left(\Sigma,\left.\mathbb{E}\right|_{\Sigma}\right) \cap \Lambda_{0}^{+}$and $z=x-y$, that is, $\boldsymbol{\beta}^{-}=\Lambda_{0}^{-}+\gamma\left(D_{0}\right)$. Hence this implies $\gamma\left(D_{0}\right)$ must be a Lagrangian subspace, because $\gamma\left(D_{0}\right)$ is always isotropic, and we have also that the closedness of the operator $\mathcal{T}_{0}^{-}$defined on $D_{0} \subset H^{1}(M, \mathbb{E})$ gives us the ellipticity of the condition for any operator $\mathcal{T}_{t}^{-}=\left(A+C_{0}\right)+\left(C_{t}-C_{0}\right)$, $t \in I$.

By the above remark, if the operator $A+C_{0}$ is invertible we have

Proposition 4.14. $\operatorname{Sf}\left\{\mathcal{T}_{s}\right\}=\operatorname{Mas}\left(\left\{\Lambda_{s}^{-}\right\}, \gamma\left(D_{0}\right)\right)$.

However we do not know whether $\operatorname{Mas}\left(\left\{\Lambda_{s}^{-}\right\}, \gamma\left(D_{0}\right)\right)$ coincides with $\operatorname{Mas}\left(\left\{\Lambda_{s}^{-} \oplus \Lambda_{0}^{+}\right\}, \delta\right)$ without the assumption of the product form near $\Sigma$. 


\section{Appendix}

In this appendix we explain the space $\mathcal{U}_{\mathcal{F}}\left(H_{J}\right)$ in the framework of the complexified symplectic Hilbert space (Proposition A.3) and give a proof for the isomorphisms:

Proposition A.1. $\pi_{1}\left(\mathcal{U}_{\lambda}\left(H_{J}\right)\right) \underset{\left(\mathcal{S}_{\lambda}\right)_{*}}{\stackrel{\sim}{\longrightarrow}} \pi_{1}\left(\mathcal{U}_{\mathcal{F}}\left(H_{J}\right)\right) \stackrel{\sim}{\longrightarrow} \mathbb{Z}$.

Let $H$ be a separable symplectic Hilbert space with the symplectic form $\omega$, an inner product $\langle\cdot, \cdot\rangle$ and an almost complex structure $J$. We assume that all of these three are compatible as before, that is,

$$
\omega(x, y)=\langle J(x), y\rangle, \quad J^{2}=-\mathrm{Id} .
$$

Let $\mathcal{L}^{\mathbb{C}}(H \otimes \mathbb{C})$ be the space of complex Lagrangian subspace in $H \otimes \mathbb{C}$ :

$$
\mathcal{L}^{\mathbb{C}}(H \otimes \mathbb{C})=\left\{l \mid l \text { is a subspace such that } l^{\perp}=J(l)\right\} .
$$

Then the subgroup of the unitary operators in $H \otimes \mathbb{C}$, denoted by $\mathcal{U}_{0}(H \otimes \mathbb{C})$, consisting of those operators $U$ such that $U(l)^{\perp}=J(U(l))$ for any $l$ in $\mathcal{L}^{\mathbb{C}}(H \otimes \mathbb{C})$ acts on $\mathcal{L}^{\mathbb{C}}(H \otimes \mathbb{C})$ transitively. This condition for $U \in \mathcal{U}_{0}(H \otimes \mathbb{C})$ is equivalent to the statement that it commutes with the almost complex structure $J$ (notice that $J$ should be considered to be complexified).

Taking the complexification of $\lambda \in \mathcal{L}(H)$ gives us a natural embedding $\mathcal{L}(H) \rightarrow$ $\mathcal{L}^{\mathbb{C}}(H \otimes \mathbb{C})$, and its restriction to $\mathcal{F} \mathcal{L}_{\lambda}(H)$ has the image in $\mathcal{F} \mathcal{L}_{\lambda \otimes \mathbb{C}}^{\mathbb{C}}(H \otimes \mathbb{C})$, a subspace of $\mathcal{L}^{\mathbb{C}}(H \otimes \mathbb{C})$ consisting of those subspaces which are Fredholm pairs with $\lambda \otimes \mathbb{C}$. We denote this map by $\mathcal{C}$.

When we consider an operator $U \in \mathcal{U}\left(H_{J}\right)$ as a real operator and take its complexification, we denote it by $U^{\mathbb{C}}$, then $U^{\mathbb{C}}$ is in $\mathcal{U}_{0}(H \otimes \mathbb{C})$ and we have $U(\mu) \otimes$ $\mathbb{C}=U^{\mathbb{C}}(\mu \otimes \mathbb{C}), \mu \in \mathcal{L}(H)$.

Let $E_{ \pm}=\{z \in H \otimes \mathbb{C} \mid J(z)= \pm \sqrt{-1} z\}$, then we have an orthogonal decomposition of $H \otimes \mathbb{C}$ as

$$
H \otimes \mathbb{C}=E_{+} \oplus E_{-},
$$

and if $U \in \mathcal{U}_{0}(H \otimes \mathbb{C})$, then $U\left(E_{ \pm}\right)=E_{ \pm}$. Hence we have an isomorphism

$$
\mathcal{U}_{0}(H \otimes \mathbb{C}) \cong \mathcal{U}\left(E_{+}\right) \times \mathcal{U}\left(E_{-}\right),
$$

where $\mathcal{U}\left(E_{+}\right)$denotes the group of unitary operators on $E_{+}$, and so on. Also the space $\mathcal{L}^{\mathbb{C}}(H \otimes \mathbb{C})$ is identified with the space of graphs of unitary transformation $U \in \mathcal{U}\left(E_{+}, E_{-}\right), U: E_{+} \rightarrow E_{-}$.

Let $\mathfrak{K}: H_{J} \rightarrow E_{+}, u \mapsto u \otimes 1-J(u) \otimes \sqrt{-1}$ and $\mathfrak{k}: H_{J} \rightarrow E_{-}, u \mapsto$ $u \otimes 1+J(u) \otimes \sqrt{-1}$, be an isomorphism and an anti-isomorphism then 
Lemma A.2. The following diagram is commutative.

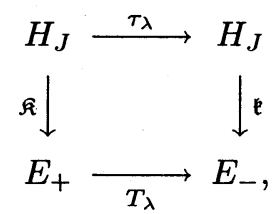

where $\tau_{\lambda}$ is the complex conjugation defined through the identification $H_{J} \cong \lambda \otimes \mathbb{C}$, and the graph of the unitary operator $T_{\lambda}$ is $\lambda \otimes \mathbb{C}=\left\{x+T_{\lambda}(x) \mid x \in E_{+}\right\}$.

Now we have

Proposition A.3. Let $\Phi: \mathcal{U}_{\mathcal{F}}\left(H_{J}\right) \rightarrow \mathcal{F L}_{\lambda \otimes \mathbb{C}}^{\mathbb{C}}(H \otimes \mathbb{C})$ be a map defined by $\Phi(V)=$ the graph of the unitary operator $-\mathfrak{k} \circ V \circ \tau_{\lambda} \circ \mathfrak{K}^{-1} \in \mathcal{U}\left(E_{+}, E_{-}\right)$. Then $\Phi$ is an isomorphism and the following diagram is commutative:

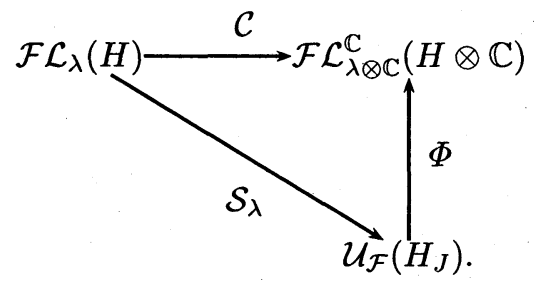

Proof. We only prove the commutativity of the diagram. Let $U \in \mathcal{U}_{\lambda}\left(H_{J}\right)$. Since $\left.U^{\mathbb{C}}\right|_{E_{ \pm}}$can be identified with $U$ through the map $\mathfrak{K}$ and $\mathfrak{k}$ respectively, we have $U^{\mathbb{C}}\left(\lambda^{\perp} \otimes \mathbb{C}\right)=\left\{U(x)-U \circ T_{\lambda}(x) \mid x \in E_{+}\right\}=\left\{x-U \circ T_{\lambda} \circ U^{-1}(x) \mid x \in E_{+}\right\}$. By the above lemma $\mathfrak{k} \circ U \circ T_{\lambda} \circ U^{-1} \circ \mathfrak{K}^{-1}=\mathfrak{k} \circ U \circ \tau_{\lambda} \circ U^{-1} \circ \tau_{\lambda} \circ \tau_{\lambda} \circ \mathfrak{K}^{-1}=$ $\mathfrak{k} \circ U \circ \theta_{\lambda}(U) \circ \tau_{\lambda} \circ \mathfrak{K}^{-1}$, which gives the commutativity of the diagram.

Let $W$ be a closed finite codimensional subspace in $\lambda \otimes \mathbb{C}$ and we denote by $\mathcal{F L}_{W}^{(0)}$ a subspace of $\mathcal{F} \mathcal{L}_{\lambda \otimes \mathbb{C}}^{\mathbb{C}}(H \otimes \mathbb{C})$ consisting of those subspaces $l$ which do not intersect with $W$. Let $H_{W}=J\left(W^{\perp} \cap(\lambda \otimes \mathbb{C})\right)+W^{\perp} \cap(\lambda \otimes \mathbb{C})$, and $\mathcal{L}\left(H_{W}\right)$ be the similar space as $\mathcal{L}\left(H \otimes \mathbb{C}\right.$ ) (note $H_{W}$ is invariant under the map $J$ ). $\mathcal{L}\left(H_{W}\right)$ is identified with the space of unitary operators on $W^{\perp} \cap(\lambda \otimes \mathbb{C})$. Let $\pi_{W}$ : $\mathcal{F} \mathcal{L}_{W}^{(0)} \ni l \rightarrow\left(l \cap\left(J\left(W^{\perp} \cap(\lambda \otimes \mathbb{C})\right)+\lambda \otimes \mathbb{C}\right)+W\right) \cap W^{\perp} \in \mathcal{L}\left(H_{W}\right)$, and then $\pi_{W}: \mathcal{F} \mathcal{L}_{W}^{(0)} \rightarrow \mathcal{L}\left(H_{W}\right)$ is a fiber bundle with contractible fibers. A typical fiber $=\pi_{W}^{-1}\left(J\left((\lambda \otimes \mathbb{C}) \cap W^{\perp}\right)\right)$ is isomorphic to the space $\widehat{\mathcal{B}}(W) \times \mathcal{B}\left(W,(\lambda \otimes \mathbb{C}) \cap W^{\perp}\right)$, where $\widehat{\mathcal{B}}(W)$ is the space of selfadjoint operators on $W$ and $\mathcal{B}\left(W,(\lambda \otimes \mathbb{C}) \cap W^{\perp}\right)$ is the space of bounded operators from $W$ to $(\lambda \otimes \mathbb{C}) \cap W^{\perp}$. Unfortunately for any pair of such subspaces $W_{1}$ and $W_{2}$ satisfying $W_{1} \subset W_{2}$ there are no natural map 
$\mathcal{L}\left(H_{W_{2}}\right) \rightarrow \mathcal{L}\left(H_{W_{1}}\right)$ which makes the diagram

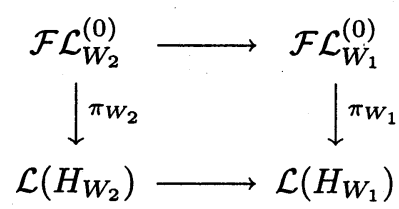

commutative. However if we define a map $s_{W}: \mathcal{L}\left(H_{W}\right) \rightarrow \mathcal{F} \mathcal{L}_{W}^{(0)}(H \otimes \mathbb{C})$ by $s_{W}(l)=l+J(W)$, then $\pi_{W} \circ s_{W}=$ Id and we have the following commutative diagram:

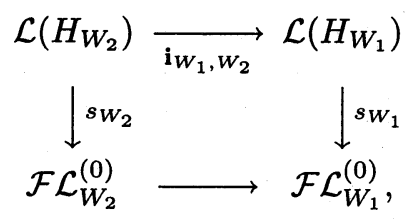

where the map $\mathbf{i}_{W_{1}, W_{2}}: \mathcal{L}\left(H_{W_{2}}\right) \rightarrow \mathcal{L}\left(H_{W_{1}}\right)$ is defined as $\mathbf{i}_{W_{1}, W_{2}}(l)=l+J\left(W_{2} \cap\right.$ $\left.W_{1}^{\perp}\right)$.

Then for any compact subset $K$ in $\mathcal{F L}_{\lambda \otimes \mathbb{C}}^{\mathbb{C}}(H \otimes \mathbb{C})$ we can find such a finite codimensional subspace $W$ in $\lambda \otimes \mathbb{C}$ that for any $l$ in $K, l \cap W=\{0\}$, so $\bigcup \mathcal{F} \mathcal{L}_{W}^{(0)}=$ $\mathcal{F L}_{\lambda \otimes \mathbb{C}}^{\mathbb{C}}(H \otimes \mathbb{C})$. Hence $\lim _{W \rightarrow\{0\}} \pi_{k}\left(\mathcal{F} \mathcal{L}_{W}^{(0)}\right)=\pi_{k}\left(\mathcal{U}_{\mathcal{F}}\left(H_{J}\right)\right)$. These facts show that the homotopy groups of $\mathcal{U}_{\mathcal{F}}\left(H_{J}\right)$ coincide with the stable homotopy groups of unitary groups, which together gives the proof of Proposition A.1.

\section{References}

[APS] M.F. Atiyah, V.K. Patodi and I. M. Singer, Spectral asymmetry and Riemannian geometry: I, Math. Proc. Cambridge Philos. Soc., 77 (1975), 43-69. II, Math. Proc. Cambridge Philos. Soc., 78 (1975), 405-432. III, Math. Proc. Cambridge Philos. Soc., 79 (1976), 71-99.

[AS] M.F. Atiyah and I.M. Singer, Index theory for skew-adjoint Fredholm operators, Inst. Hautes Études Sci. Publ. Math., 37 (1969), 5-26.

[BF1] B. Booss-Bavnbek and K. Furutani, The Maslov index - a functional analytical definition and the spectral flow formula, Tokyo J. Math., 21 (1998), 1-34.

[BF2] B. Booss-Bavnbek and K. Furutani, Symplectic functional analysis and spectral invariants, in: B. Booss-Bavnbek, K.P. Wojciechowski (eds.), "Geometric Aspects of Partial Differential Equations", Amer. Math. Soc. Series, Contemporary Mathematics, Vol. 242, Providence, R.I., 1999, pp. 53-83.

[BFO] B. Booss-Bavnbek, K. Furutani and N. Otsuki, Criss-cross reduction of the Maslov index and a proof of the Yoshida-Nicolaescu Theorem, to appear in Tokyo J. Math., 24-1 (2001).

[BW] B. Booss-Bavnbek and K.P. Wojciechowski, Desuspension of splitting elliptic symbols, Part II, Ann. Global Anal. Geom., 4 (1986), 349-400.

[CLM1] S.E. Cappell, R. Lee and E.Y. Miller, On the Maslov index, Comm. Pure Appl. Math., 47 (1994), 121-186. 
[CLM2] S.E. Cappell, R. Lee, and E.Y. Miller, Selfadjoint elliptic operators and manifold decompositions Part I: Low eigenmodes and stretching, Comm. Pure Appl. Math., 49 (1996), 825-866. Part II: Spectral flow and Maslov index, Comm. Pure Appl. Math., 49 (1996), 869-909. Part III: Determinant line bundles and Lagrangian intersection, Comm. Pure Appl. Math., 52 (1999), 543-611.

[CP] A. Carey and J. Phillips, Unbounded Fredholm Modules and Spectral Flow, Canad. J. Math., 50-4 (1998), 673-718.

[DK] M. Daniel and P. Kirk, with an appendix by K.P. Wojciechowski, A general splitting formula for the spectral flow, Michigan Math. J., 46 (1999), 589-617.

[DS] N. Dunford and J. Schwartz, Linear Operators, Part II: Spectral Theory, Interscience Publishers, 1963.

[Du] J.J. Duistermaat, On the Morse index in variational calculus, Adv. in Math., 21 (1976), 173-195.

[Fl] A. Floer, A relative Morse index for the symplectic action, Comm. Pure Appl. Math., 41 (1988), 393-407.

[FO] K. Furutani and N. Otsuki, Spectral flow and intersection numbers, J. Math. Kyoto Univ., 33 (1993), 261-283.

[Ge] E. Getzler, The odd Chern character in cyclic homology and spectral flow, Topology, 32 (1993), 489-507.

[Go1] M. de Gosson, La définition de l'indice de Maslov sans hypothèse de transversalité, C. R. Acad. Sci. Paris, 310, Série I (1990), 279-282.

[Go2] M. de Gosson, The structure of $q$-symplectic geometry, J. Math. Pures Appl., 71 (1992), 429-453.

[Ho1] L. Hörmander, Pseudo-differential operators and non-elliptic boundary problems, Ann. of Math., 83 (1966), 129-209.

[Ho2] L. Hörmander, The Analysis of Linear Partial Differential Operators III, Springer, Berlin, 1985.

[Ka] T. Kato, Perturbation Theory for Linear Operators, 2nd ed., Springer-Verlag, Berlin, 1980.

[KL] P. Kirk and M. Lesch, The $\eta$-invariant, Maslov index, and spectral flow for Dirac-type operators on manifolds with boundary, (preprint).

[Le] J. Leray, Analyse Lagrangiénne et mécanique quantique: Une structure mathématique apparentée aux développements asymptotiques et à l'indice de Maslov, Série Math. Pure et Appl., I.R.M.P., Strasbourg, 1978 (English translation 1981, MIT Press).

[Ni] L. Nicolaescu, The Maslov index, the spectral flow, and decomposition of manifolds, Duke Math. J., 80 (1995), 485-533.

[OF] N. Otsuki and K. Furutani, Spectral flow and Maslow index arising from Lagrangian intersections, Tokyo J. Math., 14 (1991), 135-150.

[Ph] J. Phillips, Self-adjoint Fredholm operators and spectral flow, Canad. Math. Bull., 39 (1996), 460-467.

[Ra] J.V. Ralston, Deficiency indices of symmetric operators with elliptic boundary conditions, Comm. Pure Appl. Math., 23 (1970), 221-232.

[RS] J. Robbin and D. Salamon, The Maslov index for paths, Topology, 32 (1993), 827-844.

[So] J.M. Souriau, Construction explicite de l'indice de Maslov, in: "Group Theoretical Methods in Physics", Springer Lecture Notes in Physics, Vol. 50, Berlin, 1975, pp. 117-148.

[Ta] C.H. Taubes, Casson's invariant and gauge theory, J. Differential Geom., 31 (1990), 547599.

[Yo] T. Yoshida, Floer homology and splittings of manifolds, Ann. of Math., 134 (1991), 277323. 
Kenro Furutani Department of Mathematics Faculty of Science and Technology ScIence University OF TOKYo 2641 NODA, CHIBA 278-8510 JAPAN E-mail: furutani@ma.noda.sut.ac.jp

Nobukazu Otsuki Department of Mathematics Faculty of Science and Technology SCIENCE UNIVERSITY OF TOKYO 2641 NODA, CHIBA 278-8510 JAPAN E-mail: nobukazu@ma.noda.sut.ac.jp 